\title{
IMPORTANCIA DE LOS PROCESOS DE VALIDACIÓN TOPOLÓGICA EN LA GESTIÓN DE ALTERACIONES CATASTRALES ${ }^{1}$
}

\author{
José Tomás Navarro Carrión ${ }^{2}$ y Alfredo Ramón Morte ${ }^{3}$ \\ Laboratorio de Geomática. Instituto Interuniversitario de Geografía ${ }^{2}$ \\ Departamento de Análisis Geográfico Regional y Geografía Física ${ }^{3}$ \\ Universidad de Alicante
}

\section{RESUMEN}

Las tecnologías de la información y la comunicación están consiguiendo que la información geográfica sea asequible a un mayor numero de profesionales a través de las Tecnologías de la Información Geográfica. La intervención multidisciplinar en el territorio enriquece la investigación y las formas de aplicación de este tipo de recursos tecnológicos. Pero esta facilidad tecnológica puede suponer el riesgo de un uso inadecuado, por falta de conocimientos técnicos adecuados a la complejidad de la información geográfica o por el mal uso de las aplicaciones informáticas.

El trabajo catastral puede beneficiarse mucho del empleo de estas tecnologías de información geográfica, al facilitar el uso, la comunicación y su administración electrónica, pero el desconocimiento de las propiedades geométricas y topológicas de la información geográfica puede llevar a cometer errores de graves consecuencias a profesionales no especializados. En este artículo ofrecemos el resultado de la investigación del trabajo de diversos juristas y técnicos, con el objetivo de desarrollar métodos automatizados y aplicaciones informáticas que permitan a los especialistas no expertos en Cartografía usar este tipo de información con garantías de exactitud al más alto nivel, como una solución eficaz para que la información geográfica con calidad topológica enriquezca la seguridad jurídica en el tráfico inmobiliario.

Palabras clave: TIC, topología, SIG, TIG, alteraciones catastrales, notario, aplicaciones geográficas informatizadas.

\section{ABSTRACT}

\section{The importance of topological validation processes when managing changes in land property}

Information and Communications Technology (ICT) is making Geographic Information (GI) reachable to an increasing number of professionals through technology. A multidisciplinary approach to land resources assessment enriches research and encourages new implementations of this type of technological resources, but the affordability of Geographic Information Technology (GIT) may lead to misuse due to lack of knowledge about GI complexity or poor user skills when working with computer applications.

Cadastral tasks can greatly benefit from the use of GIT to empower transparent transactions and e-government, but failing to handle geometric and topological properties when dealing with GI may lead to mistakes with serious consequences for non-skilled professionals. In this article, we show results of

\footnotetext{
1 Este trabajo se ha realizado como resultados del Proyecto de Investigación Aplicada "Ramón Llull", realizado en el Laboratorio de Geomática del Instituto Interuniversitario de Geografía y el Departamento de Derecho Civil de la Universidad de Alicante bajo el patrocinio del Consejo General del Notariado y Colegio Notarial de Valencia. Además, se inscribe en el marco del proyecto de investigación «El Registro de la Propiedad como instrumento vertebrador de la información territorial; datos espaciales, metadatos y Directiva INSPIRE (II)» (DER 2011-23321), financiado por el Ministerio de Economía y Competitividad.

jt.navarro@ua.es

alfredo.ramon@ua.es
} 
a research work conducted by a team of both, legal and technical professionals, whose main goal is the development of automated methods and mapping software that allow non-experts to fully embrace GI while preserving the highest level of accuracy. We foresee this as an effective solution to encompass real estate data transactions with topologically accurate GI and, thus, as a means to enforce for legal certainty.

Keywords: ICT, topology, GIS, GIT, cadastral changes, notary, geographical computer applications

\section{INTRODUCCIÓN}

Las tecnologías de la información geográfica (TIG) están alcanzando un alto nivel de eficacia en facilitar el acceso al territorio a un variado elenco de profesionales. Esta incorporación diversa aporta enfoques de distinta naturaleza y métodos de trabajo que constituyen una fuente inagotable de innovación. Juristas o sociólogos pueden ser un gran ejemplo de este proceso, incorporando el territorio en los métodos de marketing, la gestión electrónica de procesos comerciales o administración de empresas

Sin embargo, la facilidad tecnológica puede llevar consigo el riesgo de intervención de profesionales que adolezcan de un adecuado nivel de conocimiento técnico para asumir con seguridad el uso y gestión de los datos territoriales. Para muchos de estos nuevos usuarios de las TIG es fácil confundir la sutil diferencia que existe entre datos gráficos, geográficos o topológicos; igualmente, tampoco son capaces de apreciar el contraste entre la precisión geométrica de una topología vectorial y la resolución espacial de una topología raster, con todo lo que ello implica sobre el rigor de la información territorial

Es necesario que estos nuevos perfiles profesionales que se incorporan a las TIG conozcan y tengan en cuenta aspectos geográficos y técnicos complejos, condignos a este tipo de información. El desconocimiento puede llevar a cometer serios errores de enfoque y valoración de importantes consecuencias, sobre todo en trabajos que implican la gestión de información geográfica sobre propiedades inmuebles, en los que estas cuestiones alcanzan gran relevancia, como los procesos de alteración catastral y su gestión electrónica.

La dimensión jurídica o económica de la gestión de bienes inmuebles ya es bastante complicada sin la necesidad de introducir un elemento técnico que incremente las dificultades. Por ello, en el Proyecto "Ramón Llull" los especialistas en Geografía y TIG estamos investigando, junto a juristas y técnicos, métodos de trabajo y diseño de aplicaciones informáticas que conviertan la información territorial en un elemento solucionador de problemas, automatizando al máximo los procesos técnicos con garantías de ausencia de riesgos por uso inadecuado de la información geográfica de carácter topológico.

En este artículo expondremos una solución eficaz para trabajar con información geográfica sobre propiedad partiendo de unas normas de calidad topológica indispensables. A muchos profesionales, la experiencia con Sistemas de Información Geográfica (SIG) y bases de datos geográficas (geodatabases) nos ha obligado a conocer este tipo de cuestiones en profundidad, pero el desafío conseguido ha sido la definición de un método automatizado, integrado en una aplicación informática sencilla y eficaz, diseñada para un trabajo de profesionales de distinta naturaleza, aportando a todo el proceso de tramitación electrónica de alteraciones catastrales de la seguridad técnica necesaria, como complemento de una seguridad jurídica mayor y una correcta gestión económica.

\section{COORDINAR INFORMACIÓN JURÍDICA E INFORMACIÓN GEOGRÁFICA}

Con esta finalidad principal surge el Proyecto "Ramón Llull” para dar solución técnica accesible a profesionales de distinta filiación en los problemas derivados de la duplicidad de información que impera en el sistema legal español en lo referente a transacciones de bienes inmuebles. En este sentido, históricamente vienen conviviendo dos tipos de registros: por un lado el Registro de la Propiedad, de carácter jurídico y enfocado al mantenimiento de los datos sobre titularidad y cargas; por otro el Catastro,

\footnotetext{
+ El Proyecto "Ramón Llull" se viene desarrollando en la Universidad de Alicante desde el año 2003 por parte del Laboratorio de Geomática del Instituto Interuniversitario de Geografía y el Departamento de Derecho Civil. Este proyecto de investigación, patrocinado por el Colegio Notarial de Valencia y el Consejo General del Notariado, nació con el fin de diseñar un modelo de coordinación de información planimétrica - geográfica e información jurídica en materia de bienes inmuebles, susceptible de ser implantado en la red de notarías de la Comunidad Valenciana a través de un sistema informático: www.arsmagna.es
} 
de carácter fiscal, en el que se registran las características físicas de los inmuebles, es decir, localización geográfica, geometría, dimensiones y uso. Estos dos sistemas registrales han operado hasta nuestros días de forma independiente, dando lugar a lo que podemos denominar dos circuitos estancos de información territorial que conllevan la disociación entre el valor en uso y el valor en cambio de un bien inmueble (Jiménez, A. y Ramón, A., 2008).

En este escenario, en el que no existe un sistema de información territorial integral y el coste de adquisición de datos fiables se dispara, no sólo se generan distorsiones en el comportamiento económico de una finca en el mercado, sino que se producen situaciones artificiales de indeterminación jurídica y por tanto de incertidumbre a la hora de realizar transacciones mercantiles con la propiedad inmobiliaria, lo que menoscaba derechos tan fundamentales como el del acceso a una vivienda.

El Proyecto "Ramón Llull" propone unas líneas maestras básicas que están en consonancia con las reformas introducidas por la Ley 2/2011 de Economía Sostenible en materia de actividad catastral y que supone una vía de adaptación a la Norma ISO19150 LADM (Land Administration Domain Model) en lo referido a los componentes geométricos y/o espaciales:

La definición del Catastro como base de datos de carácter principal sobre la información geográfica de los bienes inmuebles (no la única, pero si la de referencia obligada).

La necesaria utilización de los datos catastrales para la constancia de la situación y modificaciones físicas de los inmuebles en los sistemas de información jurídica.

La gestión de la información geográfica en formato digital como herramienta básica para la identificación de los bienes inmuebles y de carácter preferente frente a la información basada en datos alfanuméricos, con rigor geométrico, geográfico y topológico.

\subsection{La información sobre la propiedad y la investigación geográfica española}

Desde el punto de vista académico, el Proyecto "Ramón Llull" entronca con una amplia tradición de la investigación geográfica sobre estructura de la propiedad inmobiliaria. Uno de los principales frutos del desarrollo científico de la Geografía en España ha sido la extraordinaria difusión durante la segunda mitad del siglo XX de una corriente historiográfica especializada en el estudio de la distribución y evolución de la propiedad de la tierra como factor determinante en la configuración del paisaje, la ordenación del territorio y los usos del suelo, principalmente en el ámbito rural. Con respecto a la temática catastral, dos son las principales aportaciones de los geógrafos adscritos a esta corriente: el manejo y crítica de fuentes documentales, y el estudio de los regímenes de tenencia y propiedad de la tierra (Gil, A., 1992).

En cuanto a la utilización de documentos geográficos de carácter histórico, es reseñable el recurso permanente al 'Interrogatorio para el establecimiento de la Única Contribución' o 'Catastro de Ensenada' como fuente indispensable para la reconstrucción del entorno rural y urbano del siglo XVIII a partir de la estructura de la propiedad (Arroyo, F. y Camarero, C., 1992), aunque también se han estudiado otros documentos como el 'Cadastre' de Cataluña y Baleares, los 'Libros de Padrones y Riqueza' del reino de Valencia, el Registro de Propiedades Expropiables asociado a la Ley de Bases para la Reforma Agraria de 1932, y, como fuente diacrónica, los Registros de la Propiedad (Gil, A., 1992).

Las materias tratadas en los estudios publicados es muy variada, así como numerosos los autores: Floristán, Quirós o Gómez Mendoza se han ocupado de los procesos de desamortización entre los siglos XVI y XIX; Gil Olcina ha estudiado la propiedad señorial como factor condicionante de las estructuras de propiedad recientes; Romero González ha comparado el efecto de la desamortización en los casos valenciano y castellano sobre la base de la titularidad y los modos de explotación de la tierra; López Ontiveros y Mata Olmo se han centrado en los orígenes del latifundismo en Andalucía (Gil, A., 1992). En alguno de estos estudios, principalmente en aquellos que tienen como objetivo la reconstrucción parcelaria, seguramente se evidencian las dificultades que plantea la ausencia de una referencia espacial numérica clara a la hora de describir fincas o bienes inmuebles. De hecho, esta omisión del componente espacial de los datos de propiedad inmobiliaria conduce a deficiencias tan notorias como la discrepancia por exceso de la superficie que teóricamente ocuparían las parcelas registradas de titularidad pública o privada respecto de la superficie real que abarca el territorio peninsular español. 
En la actualidad, en el contexto de divulgación y consumo masivo de información geográfica digital, un nutrido grupo de geógrafos trabaja actualmente en la definición de nuevos usos económicos y sociales de dicha información. Esto incluye el manejo de información relativa a bienes inmuebles y evidentemente, el análisis de la distribución parcelaria enfocado principalmente a la formulación de modelos digitales dinámicos que permitan comprender mejor la evolución reciente de la propiedad y los usos del suelo. Para ello se apoyan en lo que ha dado en denominarse Geotecnología o Tecnologías de la Información Geográfica. Este proceso de tecnificación de la Geografía, que al fin y al cabo no deja de ser un reflejo del contexto socio-tecnológico en el que nos hallamos inmersos, ha suscitado muchos debates de gran calado teórico y metodológico en el seno de nuestra disciplina. Quizá la conclusión más interesante que podemos sacar es que no nos encontramos ante una ruptura epistemólogica tal y como podría esgrimirse siguiendo el modelo kuhniano de evolución científica, sino en el inicio de un nuevo ciclo marcado por el surgimiento de la Ciencia de la Información Geográfica cuya vocación no es reemplazar sino aportar innovaciones metodológicas al cuerpo teórico de la Geografía (García, M. D. y otros, 1992).

En esta nueva andadura, las Tecnologías de la Información Geográfica pueden considerarse no sólo un instrumento sino una parte integrante del núcleo de la Geografía, puesto que se asimilan metodológicamente a las cuatro tradiciones centrales de la investigación geográfica identificadas por Harvey (Chuvieco, E. y otros, 2005): el análisis morfométrico (a través de la Teledetección y los Sistemas de Posicionamiento Global), las relaciones de causalidad (mediante el análisis espacial y la geoestadística), la explicación temporal (de nuevo mediante el uso de la Teledetección) y el análisis funcional y ecológico (a través del geoprocesamiento). El impacto tecnológico sobre la Geografía tiene un efecto positivo y necesariamente integrador: ahora contamos con herramientas y técnicas de análisis que nos permiten afrontar un abanico mayor de problemas territoriales, prácticamente a cualquier escala y con independencia de los presupuestos teóricos (Chuvieco, E. y otros, 2005). Pero también existe un efecto recíproco que revaloriza la Geografía (Buzai, G. D., 1999; Chuvieco, E. y otros, 2005) en cuanto que proveedora de fundamentos y conceptos teóricos que permiten el manejo adecuado de la tecnología.

Aunque más empleadas inicialmente en la investigación de fenómenos físico-bióticos, las Tecnologías de la Información Geográfica se aplican cada vez más a la resolución de problemas tradicionales de modelado y análisis multivariado de procesos histórico-geográficos, donde la explicación temporal es clave para su comprensión (Chuvieco, E. y otros, 2005).

En esta corriente del nuevo paradigma del pensamiento geográfico se imbrica el Proyecto "Ramón Llull", conjugando instrumentos cada vez más comunes en investigación geográfica aplicada y que son cruciales a su vez para el correcto desarrollo y mantenimiento de la información catastral: la cartografía digital, las bases de datos relacionales, el análisis orientado a objetos, el análisis espacial, el geoprocesamiento, el conocimiento compartido a través de las bases de datos geográficas y el trabajo colaborativo interdisciplinar que propicia la comunicación por Internet. El Catastro al evolucionar tecnológicamente y convertirse en una base de datos geográfica, deja de ser así una mera fuente de información para el estudio geográfico del territorio, para convertirse en un fin/objetivo de la investigación geográfica y en una plataforma de comunicación de la información territorial.

\subsection{La identificación topológica de la finca como clave para la gestión de la propiedad}

El protocolo de actuación en el Proyecto "Ramón Llull" define una serie de claves que han determinado todos los desarrollos posteriores. La primera deriva del énfasis puesto en el valor jurídico de la geometría de los lindes y la planimetría, si partimos del hecho de que el Catastro es la principal fuente de información de base cartográfica de referencia obligada ${ }^{5}$. Esta integración se cumple tanto en el aspecto numérico como en el aspecto lógico, o lo que es lo mismo, la implantación de un sistema de información compatible

\footnotetext{
Esto no quiere decir que la identificación de una propiedad se tenga que iniciar siempre en la información catastral, pues ésta puede limitarse a un "marco de referencia obligado"; de hecho, la información puede y debe venir en muchos casos procedente del trabajo de campo y proceso topográfico avalado por técnicos y especialistas, pero sin olvidar que este marco catastral nos permite participar de unas normas de formatos de datos, criterios de calidad geométrica y geográfica (sistemas de coordenadas de referencia) que son necesarios para adaptarnos a una normalización que permita coordinar y compartir esfuerzos entre los distintos agentes que intervienen en el proceso.
} 
y adaptado a los requerimientos de procedimiento y formatos de la Oficina Virtual de Catastro (OVC). De esta forma, la Dirección General de Catastro podrá disponer de un servicio periférico de descarga y retorno de información que, independientemente de los agentes que intervengan en el proceso de tráfico inmobiliario, garantiza la coherencia y la conformidad en el flujo de datos geográficos catastrales.

Por otro lado, se aboga por el concepto de continuo cartográfico frente al criterio de consideración aislada de los bienes inmuebles en tanto en cuanto, para establecer su identificación, no debería bastar una referencia catastral o un código registral asociado a una polilínea de CAD, por mucha precisión técnica que se pueda alcanzar en ello. De hecho, este necesario contexto geométrico - geográfico da respuesta al problema de la duplicidad e incoherencia de información ya que, por un lado, la actualización de la descripción física de un inmueble se produce siempre en relación con sus colindantes de forma que la cartografía catastral de un territorio mantenga una cohesión perfecta entre los inmuebles que la integran, sin vacíos, solapamientos o desplazamientos entre los mismos; y por otro, se da siempre preferencia a la información gráfica frente a la alfanumérica para la determinación espacial de los bienes inmuebles, de tal forma que no puedan producirse excesos o defectos de cabida, es decir, que pueda existir contradicción entre los linderos de un inmueble y su superficie real.

El hecho de que cualquier transacción de propiedad inmobiliaria implique por norma general la aportación de documentación fehaciente y su asentamiento en escritura pública, hace que el período de tramitación de dicha escritura constituya la situación temporal óptima para determinar la revisión del bien inmueble y su actualización geográfica en el Catastro, incluso por procedimiento de administración electrónica, a través del servicio de la O.V.C. La incorporación de la información planimétrica al documento jurídico es, por tanto, otra de las claves del proyecto. La principal ventaja de este procedimiento es la seguridad jurídica: al coincidir la modificación gráfica de la finca con el otorgamiento de la escritura pública se evitan situaciones ulteriores de modificaciones de inmuebles efectuadas sin el consentimiento del titular. Por otra parte, se corrige y pone freno a las disconformidades entre la información gráfica y la alfanumérica, se hace coincidir en el tiempo el acto jurídico de la alteración catastral con la actualización de la base cartográfica de referencia y se produce un ahorro en los costes de obtención del dato geográfico.

El concepto fundamental, en el que confluyen los anteriores requerimientos, no es más que la prevalencia de la realidad sobre cualquier otro tipo de datos. El requisito de autenticidad interesa a ambos componentes de la información territorial, el físico y el jurídico. En el nivel físico y según la complejidad del problema a resolver, se requiere que un técnico competente realice la medición sobre el terreno para posteriormente apoyarla sobre la base cartográfica catastral y en el nivel jurídico, se requiere que la alteración dictaminada en el nivel físico se adapte al ordenamiento jurídico en materia de ordenación patrimonial de bienes inmuebles, cuyo principio fundamental es que ningún derecho puede ser transmitido sin el consentimiento de su titular, evitando así situaciones jurídicas de indefensión (Jiménez, A. y Ramón, A., 2008).

\section{3. ¿CÓMO GARANTIZAR LA AUTENTICIDAD FÍSICA DE UN BIEN INMUEBLE?}

El resultado tangible del Proyecto "Ramón Llull" es un sistema de intercambio de información y un modelo de uso colaborativo necesariamente complejos, puesto que son muchas las implicaciones jurídicas derivadas del trabajo de los agentes públicos, técnicos y privados que intervienen en la cadena del negocio inmobiliario. Dicha complejidad ha determinado los esfuerzos de investigación conducentes al desarrollo de una de las partes elementales de dicho modelo: la que permite garantizar la autenticidad del bien inmueble desde el punto de vista físico. En este artículo se aborda en profundidad este componente del proyecto, elemental en cuanto que constituye la base sobre la que se construyen los mecanismos de transferencia de información con Catastro o las herramientas de medición y diagnóstico específicas para notarios y técnicos competentes. En todo caso, muchas veces es una base conceptual que queda relativamente oculta a los potenciales usuarios del sistema pero cuyo interés académico desde una perspectiva cartográfica es evidente, puesto que se plasma en un modelo de validación topológica que constituye el marco regulador y de cálculo que garantiza ese ajuste entre la realidad, el levantamiento o replanteo propuesto por el especialista en el trabajo y medición de campo y la cartografía que se debe usar como referencia. 
El modelo de validación topológica del Proyecto "Ramón Llull" es un modelo orientado a objetos organizado en tres capas lógicas, tal y como ilustra la Figura 1. La información relevante para la elaboración del diagnóstico acerca de la coherencia de una operación de alteración catastral y, por tanto, los datos que determinan la autenticidad física de las fincas candidatas a formar parte de la base cartográfica de referencia, fluyen entre las distintas capas. Cada una de ellas corresponde a un grado de abstracción diferente y engloba un conjunto de clases propio.

Figura 1. Capas del modelo de validación topológica.

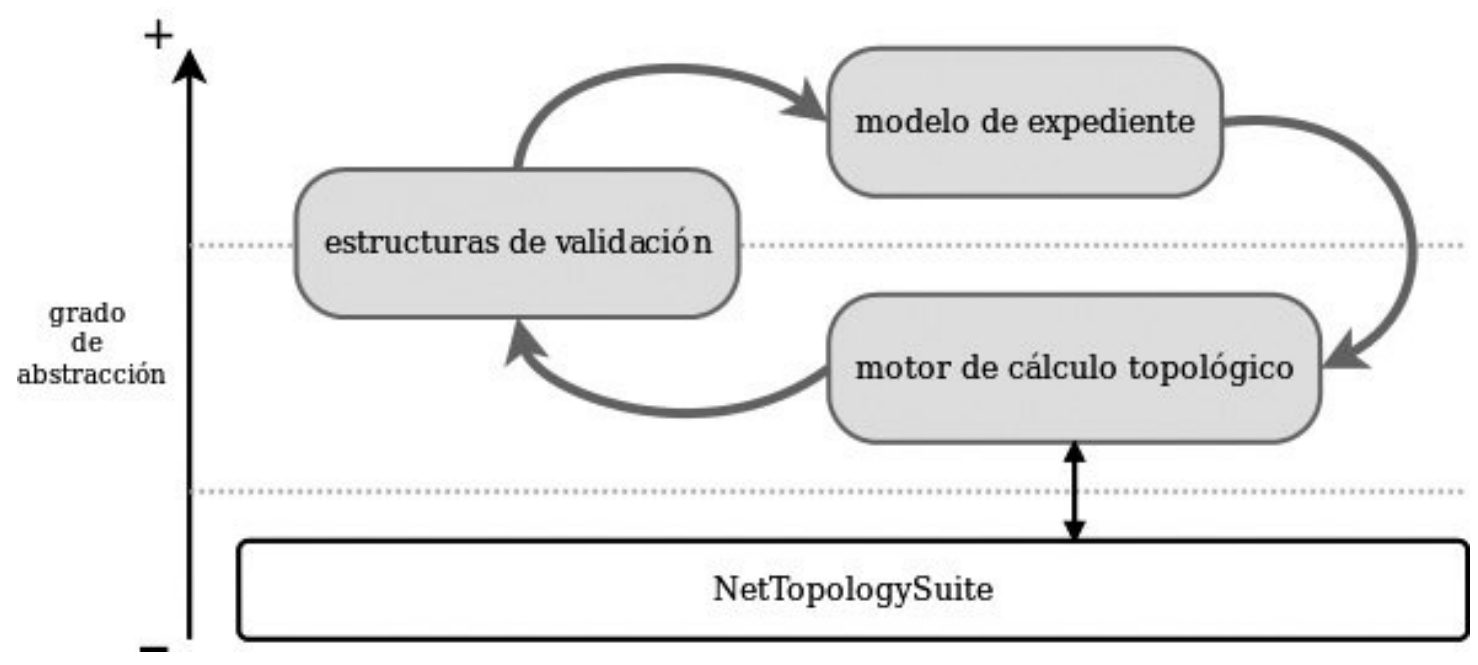

Las piezas que constituyen el modelo de validación son, por un lado, el modelo de expediente, y por otro, el motor de cálculo topológico y las estructuras de validación. El mayor grado de abstracción se encuentra en el modelo de expediente, que representa conceptos o entidades tangibles a nivel físico y administrativo tales como la parcela catastral o el propio trámite de alteración (agregación, segregación o deslinde). En este nivel lógico se inicia la ejecución de los planes de validación y en última instancia se recibe el diagnóstico. En un nivel intermedio de abstracción se encuentra el motor de cálculo topológico, que realiza operaciones básicas de poligonización, cómputo de superficies, análisis de grafos y análisis espacial mediante matrices DE-9IM ${ }^{6}$. El intercambio de datos entre estas dos capas está condicionado a un conjunto de estructuras de validación topológica, es decir, el output de un método del motor de cálculo será normalmente una determinada estructura de validación que el modelo de expediente podrá reutilizar como input a la hora de invocar algún otro método del motor de cálculo. Cada estructura de validación es una regla que contiene en sí misma tanto el resultado de la evaluación como un producto geométrico en caso de evaluación positiva. De esta forma, para cada operación de alteración catastral, se configura una secuencia lógica de reglas predefinidas que denominamos plan de validación. Cada plan de validación es un modelo de geoprocesamiento per se. Es preciso mencionar que en el nivel más bajo de abstracción se encuentra la librería de análisis espacial NetTopologySuite, de la que dependen la práctica totalidad de los métodos de cálculo del motor topológico.

\subsection{El motor topológico y las estructuras de validación de la geoinformación}

El motor de cálculo topológico del Proyecto "Ramón Llull" se organiza en torno a la clase TopologyBuilder, que define e implementa los métodos de cálculo sobre geometrías vectoriales bidimensionales necesarios para verificar la coherencia de cualquiera de las operaciones de alteración catastral del modelo de expediente.

\footnotetext{
6 El modelo o matriz DE-9IM (Dimensionally Extended nine-Intersection Model) es un modelo topológico y un estándar utilizado para describir las relaciones espaciales de dos regiones poligonales distintas y superpuestas (dos geometrías en dos dimensiones, R2) desarrollada para su uso como base para las normas de las consultas y operaciones de geoproceso de los sistemas de información geográfica (GIS) y bases de datos espaciales (geodatabases).
} 
El único método de la clase TopologyBuilder que podemos considerar independiente es CalculateArea, el cuál es además polimórfico puesto que permite el cálculo de superficies tanto a partir de una simple lista de coordenadas como de un objeto de tipo MultiLineString de la especificación Simple Features del Open Geospatial Consortium (OGC) ${ }^{7}$. El resto de métodos presenta diversos grados de dependencia funcional entre sí. Esto significa que, en la mayoría de los casos, están específicamente diseñados para operar secuencialmente de forma que el output de un método determine o incluso actúe como el input de otro, emulando como ya se ha mencionado el comportamiento de los modelos de geoprocesamiento. Este patrón de diseño permite dotar a los planes de validación de una infraestructura funcional flexible.

Figura 2. Diagrama UML del motor de cálculo topológico.

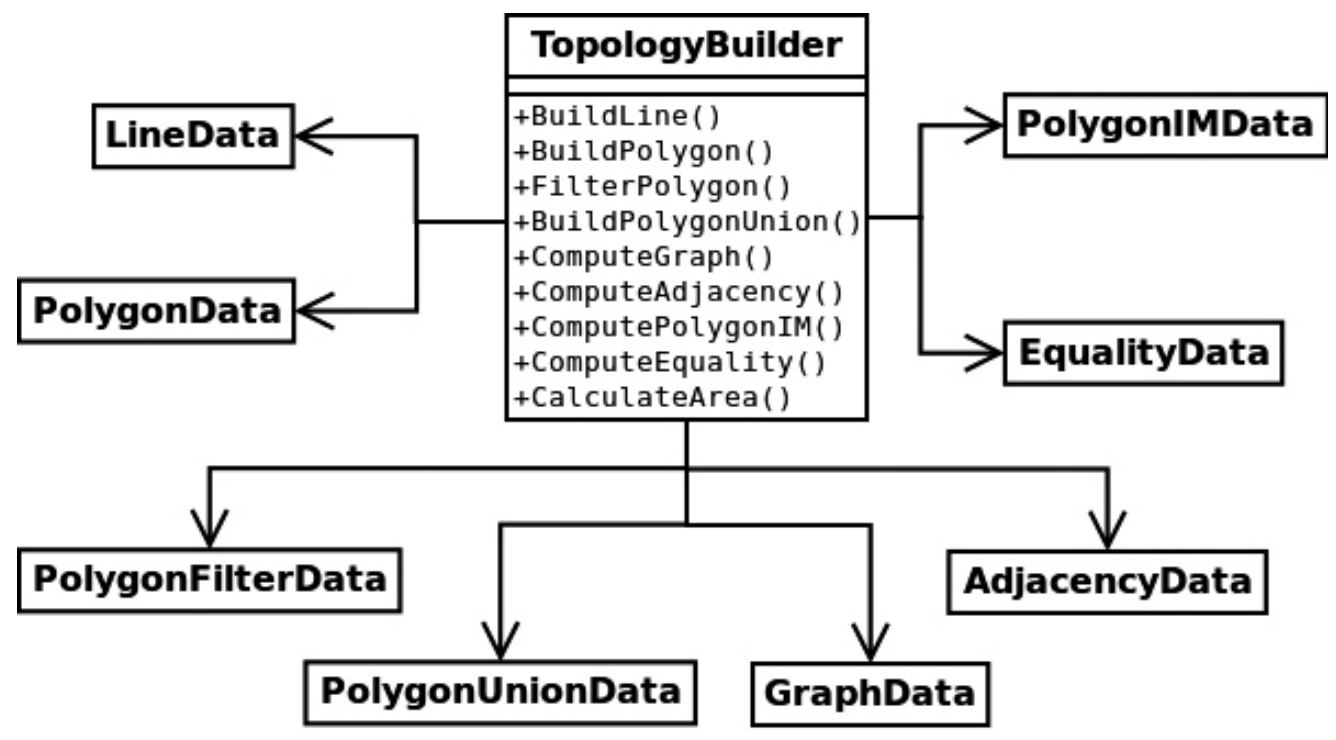

El output de un determinado cálculo topológico es muy variable en cuanto a su complejidad. En general, nos puede interesar no sólo el resultado geométrico (si es que se trata de operaciones de geoprocesamiento), sino también conservar información acerca de las geometrías que participan, evaluar la validez del cálculo, recuperar información geométrica adicional o generar mensajes inteligibles para el usuario. Por tanto, tal y como ilustra la Figura 2, cada uno de los métodos del motor topológico genera un output estructurado o estructura de validación, más o menos compleja según la naturaleza del cálculo. Los planes de validación topológica no son más que algoritmos construidos a partir de la secuenciación de dichas estructuras. A continuación se revisan de forma aislada y detallada cada una de estas estructuras de validación y los métodos que las originan.

\subsection{Creación de geometrías lineales}

La creación de objetos de tipo MultiLineString según el estándar Simple Features es la operación preliminar del motor de cálculo topológico. La Figura 3 detalla las clases que intervienen. El método BuildLine de la clase TopologyBuilder se encarga de generar dicha geometría lineal. Para ello precisa como input la lista de coordenadas del perímetro general de la parcela catastral, que se puede obtener del correspondiente fichero de intercambio FXCC. Este método necesita para su cometido del concurso de un objeto que implemente métodos concretos de transformación de listas de coordenadas a geometrías estandarizadas (GeometryBuider).

\footnotetext{
El Open Geospatial Consortium (OGC) es un consorcio creado a finales del siglo XX por la integración de diversas empresas y fundaciones de ámbito internacional, con la finalidad de fijar estándares abiertos e interoperables dentro de los Sistemas de Información Geográfica y el WEB, buscando acuerdos entre empresas del sector para facilitar la interoperabilidad de los datos geográficos, su geoproceso y el intercambio de los mismos entre la cada vez mayor cantidad de usuarios de geoinformación
} 
Figura 3. Diagrama UML de la generación de geometrías lineales.

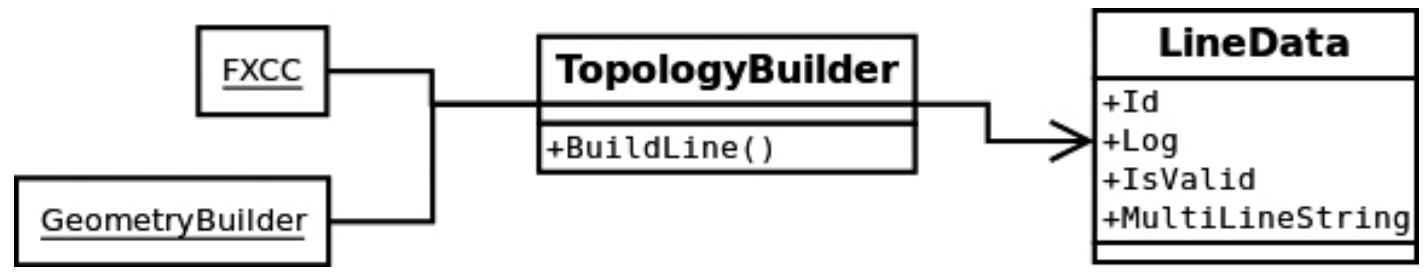

El output del método BuildLine es una estructura de validación de tipo LineData, que almacena el identificador de la parcela (propiedad Id), un texto con información acerca de posibles incidencias en la creación de la geometría lineal (propiedad Log), el resultado positivo o negativo de la transformación (propiedad IsValid) y la geometría de salida (propiedad MultiLineString). La transformación de las coordenadas del FXCC en un objeto MultiLineString no tiene implicaciones a nivel topológico (en ambos casos se trata de estructuras de tipo spaghetti). Se trata simplemente de un traspaso necesario de información geométrica hacia el ámbito operativo de la librería NetTopologySuite.

\subsection{Creación de geometrías poligonales}

El proceso básico del motor de cálculo topológico es la poligonización. En el ámbito del Proyecto "Ramón Llull" este proceso está determinado por la especificación Simple Features del OGC y se define como la obtención de un objeto de tipo Polygon a partir del perímetro general de una parcela, previamente almacenado como objeto MultiLineString. Actualmente el modelo de validación contempla que un contorno de parcela delimita un único polígono simple, es decir, no se toman en consideración huecos interiores a la parcela ni geometrías discontiguas (MultiPolygon).

Utilizando internamente el gestor de poligonización de NetTopologySuite, el método BuildPolygon de la clase TopologyBuilder implementa este proceso a partir de una estructura LineData, y vuelca el resultado en una nueva estructura PolygonData, tal como se aprecia en la Figura 4. Los requisitos para que la poligonización pueda efectuarse son:

Que la geometría lineal se haya construido correctamente, lo cual se evalúa a partir de la propiedad IsValid de la estructura LineData.

Que no existan inconsistencias topológicas tales como líneas inacabadas, líneas sobreacabadas o contornos inválidos. El propio gestor de poligonización de NetTopologySuite proporciona dicha información.

Figura 4. Diagrama UML de la generación de geometrías poligonales.

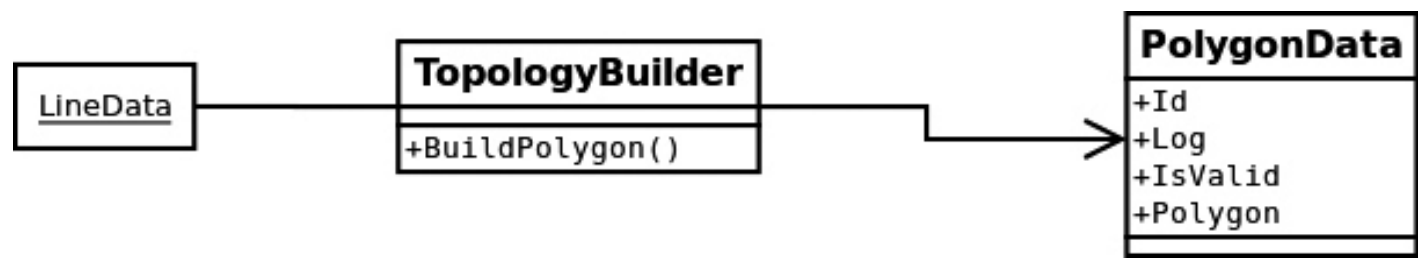

La estructura PolygonData resultante almacena el identificador de la parcela cuyo perímetro se poligoniza (porpiedad Id), la validez de la poligonización (propiedad IsValid), información legible respecto a la no conformidad con alguno de los requisitos (propiedad $\log$ ) y la geometría poligonal de salida (propiedad Polygon).

\subsection{Análisis espacial mediante matrices DE-9IM}

El análisis comparativo de pares de geometrías poligonales mediante matrices DE-9IM es uno de los pilares del modelo de validación topológica del Proyecto "Ramón Llull", tanto por la flexibilidad del predicado Relate como por la posibilidad de evaluar en cada comparación todos los predicados que sean susceptibles de participar en el contexto de cualquier plan de validación. 
El encargado del proceso es el método ComputePolygonIM de la clase TopologyBuilder, y su estrategia se basa en el cálculo de las $5 n-1$ posibles matrices DE-9IM de una lista de objetos Polygon de entrada, siendo $n$ el tamaño de dicha lista. Como ilustra la Figura 5, el input es un conjunto de estructuras PolygonData, mientras que el output es un conjunto de estructuras PolygonIMData, una por cada matriz DE-9IM. En principio el único requisito para proceder al cálculo de una matriz DE-9IM es que el par de estructuras PolygonData participantes sean válidas, y por tanto hagan referencia a dos geometrías poligonales correctas.

Figura 5. Diagrama UML del análisis mediante matrices DE-9IM.

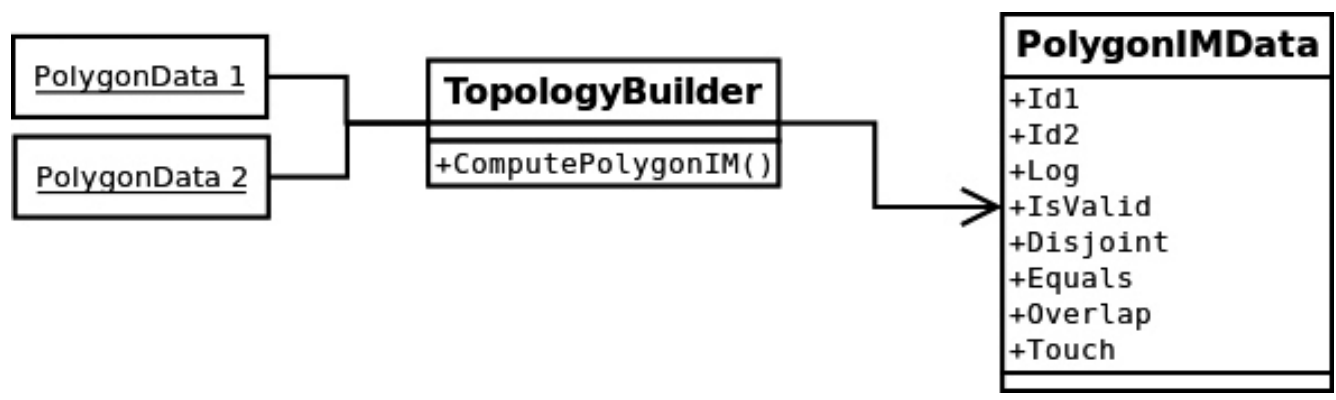

Cada estructura PolygonIMData de salida almacena los identificadores de las dos parcelas cuyos perímetros se han comparado (propiedades Id1 e Id2). Normalmente la estructura es válida (propiedad IsValid) si el par de polígonos de entrada es válido. En caso contrario o ante cualquier otra contingencia, la estructura puede proporcionar un texto explicativo de la incidencia (propiedad Log). Con todo, la verdadera utilidad de la estructura PolygonIMData radica no obstante en el conjunto de las cuatro propiedades booleanas (Disjoint, Equal, Overlap y Touch) que cualifican la relación espacial entre los polígonos de entrada. La Tabla 1 detalla la metodología de evaluación de la matriz DE-9IM resultante que determina la asignación de cada una de estas propiedades a true o false.

Tabla 1. Tipos de evaluación de la matriz DE-9IM almacenados en la estructura de validación PolygonIMData.

\begin{tabular}{|c|c|c|c|}
\hline propiedad & predicado & patrón & ejemplo \\
\hline $\begin{array}{c}\text { Disjoint } \\
\text { (disjunción) }\end{array}$ & $\begin{array}{c}\text { Disjoint } \\
\text { (predefinido) }\end{array}$ & no aplica \\
\hline $\begin{array}{c}\text { Equal } \\
\text { (coincidencia) }\end{array}$ & Relate & FF2F11212 \\
\hline $\begin{array}{c}\text { Overlap } \\
\text { (desbordamiento } \\
\text { parcial) }\end{array}$ & Relate & 212111212 & \\
\hline $\begin{array}{c}\text { Overlap } \\
\text { (desbordamiento } \\
\text { total) }\end{array}$ & Relate & 212101212 & \\
\hline $\begin{array}{c}\text { Touch } \\
\text { (adyacencia) }\end{array}$ & Relate & 2 FFF1FFF2 \\
\hline
\end{tabular}

\subsection{Evaluación de la regla de adyacencia}

Dado un conjunto de objetos de tipo Polygon, la regla de adyacencia es un método simplificado que permite discernir de forma agregada si dicho conjunto forma un continuo, es decir, un mosaico en el que no existen elementos totalmente disjuntos del resto ni elementos superpuestos a otros. La evaluación de esta regla para un conjunto de polígonos puede expresarse como: 


$$
\exists\left(\sum(T) \geqslant \sum(P)-1\right) \wedge \sum(O)=0
$$

Así pues, la regla se cumple sólo si se dan dos condiciones:

1. Que la suma de adyacencias entre polígonos $\Sigma(T)$ sea mayor o igual al total de polígonos en el conjunto $\Sigma(P)$ menos uno.

2. Que la suma de superposiciones entre polígonos $\Sigma(O)$ sea cero.

La evaluación de la regla de adyacencia es directa cuando partimos de una lista de estructuras PolygonIMData, ya que basta con calcular el total de adyacencias y el total de superposiciones mediante el análisis de las propiedades booleanas Touch y Overlap de cada estructura componente. Esta lista constituye el input del método ComputeAdjacency de la clase TopologyBuilder, que vuelca a su vez el resultado en una nueva estructura de validación de tipo AdjacencyData, tal como se ilustra en la Figura 6. Dicha estructura sólo proporciona información acerca del cumplimiento de la regla de adyacencia (propiedad IsValid) y un mensaje justificativo en caso de no conformidad (propiedad Log). La estructura AdjacencyData causa la resolución de un plan de validación cuando la propiedad IsValid está asignada a false.

Figura 6. Diagrama UML de la evaluación de la regla de adyacencia.

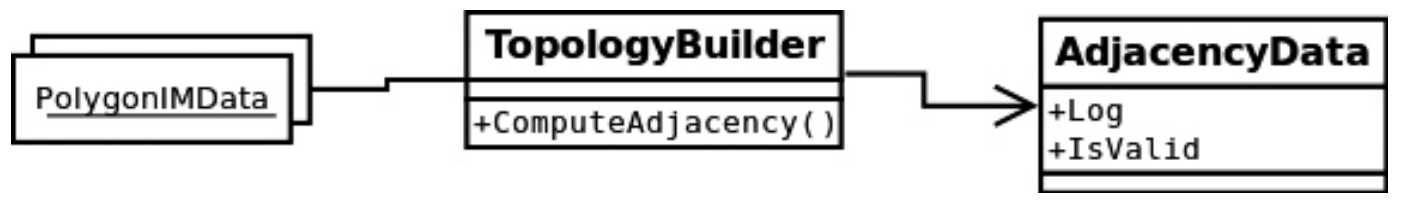

\subsection{Unión de polígonos}

La unión de polígonos es uno de los procesos topológicos y de geoprocesamiento más comunes. En el caso del Proyecto "Ramón Llull" es clave para evaluar la coherencia de cualquier alteración que implique conjuntos de parcelas de entrada (agregaciones) o de salida (segregaciones y divisiones).

Como se ve en la Figura 7, el método BuildPolygonUnion de la clase TopologyBuilder es el responsable del cálculo. Utiliza como input una lista de estructuras PolygonData, cada una de las cuales hace referencia mediante la propiedad Polygon al polígono formado por el perímetro general de una parcela. El resultado se vuelca en una nueva estructura de tipo PolygonUnionData que hereda los identificadores de cada parcela componente (propiedad Ids), informa sobre la validez del cálculo (propiedad IsValid), proporciona una descripción de la incidencia que haya podido causar la suspensión del proceso (propiedad Log) y, en caso de éxito, almacena la geometría poligonal resultante (propiedad Polygon).

Figura 7. Diagrama UML de la unión de polígonos.

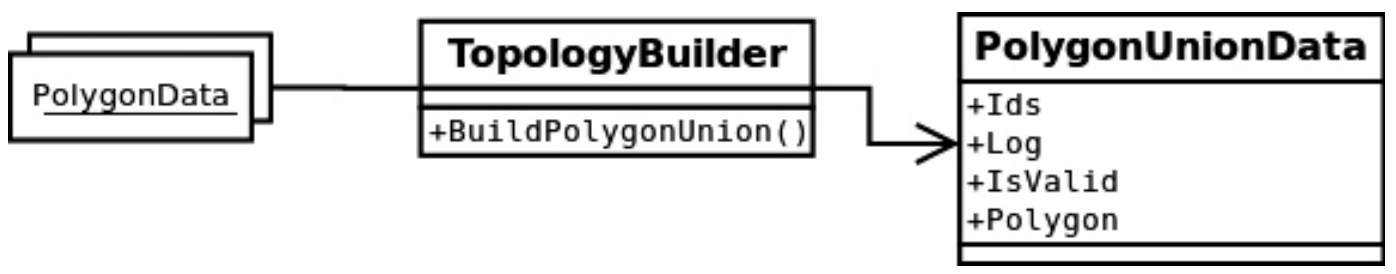

El objetivo del método BuildPolygonUnion es construir una única geometría de tipo Polygon con una única línea perimetral externa. Para conseguirlo, no parte del conocimiento previo acerca de la secuencia ordenada de adyacencias, sino que utiliza una estrategia de evaluación doble:

De forma previa al proceso se comprueba si cada estructura PolygonData entrante es válida. Una estructura no válida suspende el proceso e invalida a su vez la estructura PolygonUnionData de salida.

Con posterioridad al proceso se analiza si la geometría resultante es de tipo Polygon. En caso negativo también se invalida la estructura PolygonUnionData de salida. Un ejemplo claro lo tenemos en el caso de polígonos de entrada totalmente disjuntos del resto, con lo que el resultado de la unión sería un geometría de tipo MultiPolygon. 
El proceso de unión propiamente dicha se apoya en la infraestructura lógica de NetTopologySuite, cuyo enfoque es incremental. Para ello creamos un objeto de tipo Geometry que hace las funciones de memoria intermedia y al que vamos agregando cada polígono del input en el mismo orden en el que aparece en la lista. Previamente a su incorporación a la memoria intermedia, cada polígono sufre el descarte de cualquier línea perimetral interior. Evidentemente esto implica realizar tantas llamadas al método Union del objeto Geometry como polígonos haya en la lista de entrada, lo que constituye una implementación conforme con la especificación Simple Features. En última instancia, es sobre este objeto Geometry (un tipo abstracto) sobre el que realizamos la evaluación de postproceso: la comprobación del tipo concreto de la geometría final y la invalidación de la estructura en caso de que no se trate de un objeto de tipo Polygon.

\subsection{Construcción de grafos}

Un grafo, desde el punto de vista de la topología vectorial, es aquella estructura de datos que permite almacenar un conjunto de geometrías así como las relaciones espaciales entre los distintos elementos que componen dichas geometrías. Esto implica registrar no sólo posiciones en el espacio sino también su orden o secuencia, así como las relaciones derivadas de conectividad y adyacencia. La estructura empleada en el Proyecto "Ramón Llull" es la de arco-nodo tal como la implementa NetTopologySuite.

Figura 8. Ejemplo de grafo topológico con nodos de grado 3.

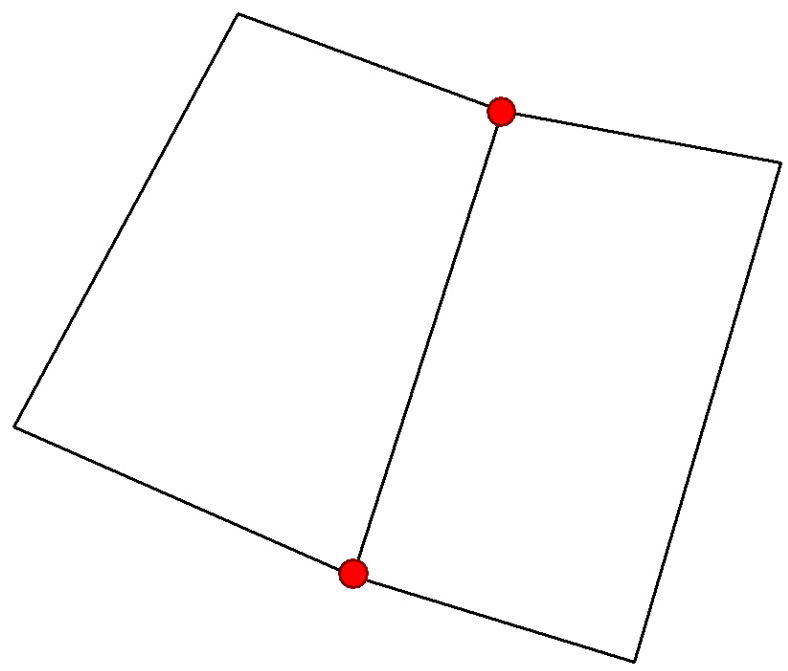

En esta versión del modelo de validación, el objetivo de la generación de grafos no es su almacenamiento para una utilización posterior, sino la identificación de los nodos de grado 3, es decir, aquellos en los que confluyen tres arcos, tal y como se muestra en la Figura 8. Estos nodos son importantes ya que hacen referencia a vértices en los que existe la posibilidad de que se haya producido un truncamiento y por tanto pueden indicar una distorsión perimetral no controlada, incidencia cuyo tratamiento se detalla más adelante.

Como puede apreciarse en la Figura 9, el método ComputeGraph de la clase TopologyBuilder es el responsable de construir un grafo a partir de un conjunto de estructuras LineData. Concretamente, el input que se le proporciona al motor de generación de grafos de NetTopologySuite es el conjunto de perímetros generales de parcela formado por cada uno de los objetos de tipo MultiLineString a los que se hace referencia en la propiedad MultiLineString de cada estructura LineData de entrada. El output es una estructura de tipo GraphData que almacena los identificadores de las parcelas cuyos perímetros generales han intervenido en el grafo (propiedad Ids), la validez del proceso y un mensaje explicativo en caso de incidencia (propiedades IsValid y Log respectivamente). En principio para que la estructura GraphData sea válida y el grafo se genere correctamente, el único requisito es que las estructuras LineData que intervienen también lo sean. Para terminar, la lista de nodos de grado 3 presentes en el grafo queda referenciada por la propiedad Degree3Nodes. 
Figura 9. Diagrama UML de la construcción de grafos.

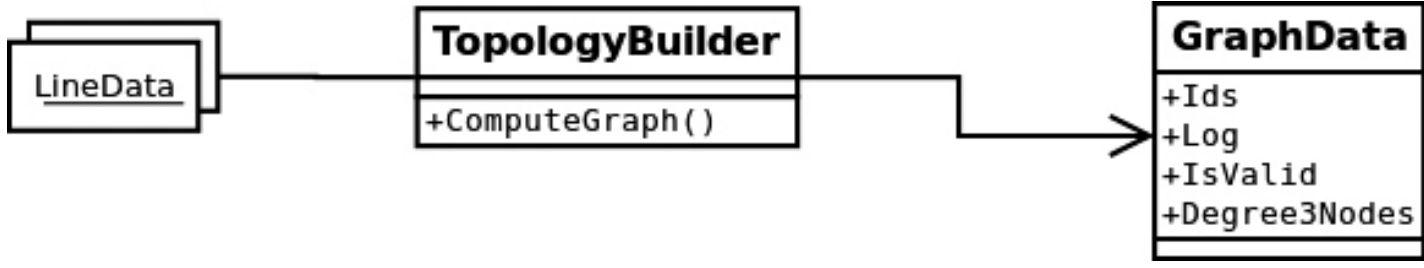

La estrategia de construcción de un grafo en NetTopologySuite es en parte similar a la de la unión de polígonos. Se necesita un objeto de tipo MultiLineString que actúe de memoria intermedia. Mediante sucesivas llamadas al método Union de dicho objeto, se van agregando los distintos MultiLineString de cada estructura LineData de entrada. Una vez completado el MultiLineString de unión debemos descomponerlo en sus elementos lineales básicos (LineString). Y por último, cada uno de estos elementos de tipo LineString se añade al grafo como un arco.

\subsection{Detección de vértices truncados}

El truncamiento es un efecto inevitable en cualquier proceso de intersección de geometrías lineales cuando las coordenadas están basadas en un modelo de precisión no exacto, como es el caso de la mayor parte de sistemas informáticos, que suelen emplear como máximo el modelo de doble precisión, que permite representaciones numéricas con 16 dígitos de precisión aproximadamente. La consecuencia es que, aunque no se produzca un redondeo explícito, el punto calculado para una intersección de dos líneas no ortogonales es normalmente una aproximación al punto exacto, tal y como se ilustra en la Figura 10.

En lo que concierne a las alteraciones catastrales, toda segregación o división parcelaria es susceptible de generar este tipo de distorsión geométrica. El modelo de validación del Proyecto "Ramón Llull" se ocupa únicamente de la detección y filtrado de los vértices truncados que hayan podido generarse en la proximidad del perímetro de la parcela original, de forma que no intervengan en el proceso de diagnóstico del plan de validación. La Figura 11 muestra cómo a partir de los nodos de grado 3 del grafo (véase la Figura 8), un perímetro general $P$ y un perímetro derivado $P 1$, pueden aislarse los candidatos a vértice truncado producto del cálculo de la intersección entre $P$ y una línea divisoria $D$.

Figura 10. Truncamiento de puntos calculados de intersección en un modelo de precisión no exacto.
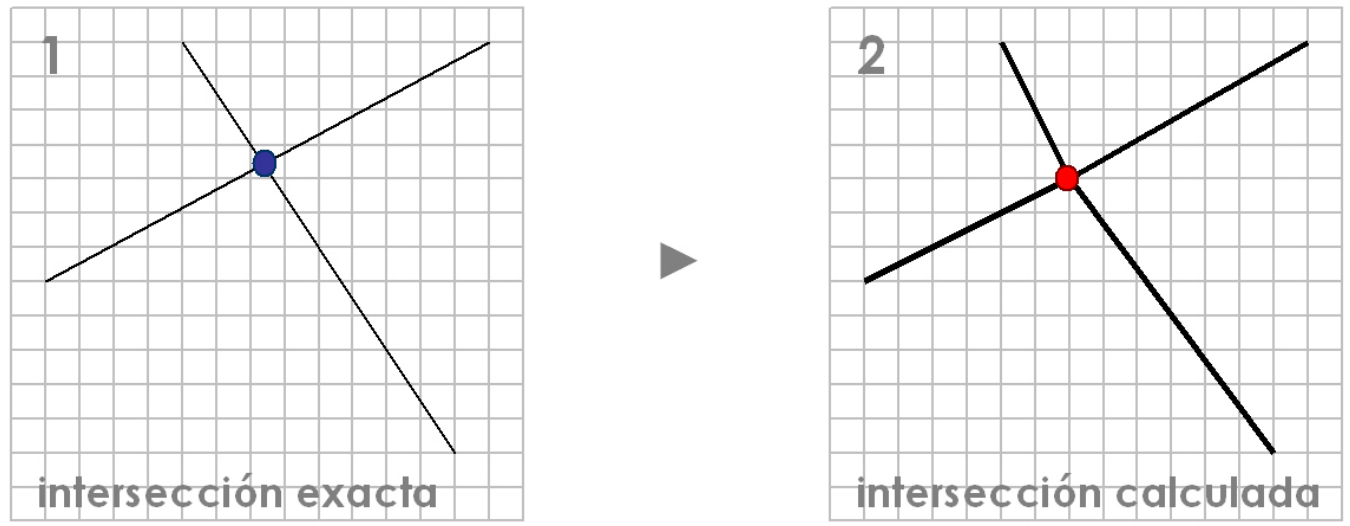

El método FilterPolygon de la clase TopologyBuilder (véase Figura 12) implementa el algoritmo de filtrado de vértices truncados a partir de tres inputs:

Una estructura de tipo GraphData que aporta las coordenadas de los candidatos a vértice truncado (propiedad Degree3Nodes).

Una estructura de tipo PolygonUnionData que proporciona el polígono a filtrar, es decir, aquel que se forma mediante la unión de las parcelas participantes en el grafo (propiedad Polygon) y es susceptible de 
contener vértices truncados. Por tanto, la lista de identificadores de parcela (propiedad Ids) coincide en ambas estructuras, GraphData y PolygonUnionData.

Una estructura de tipo PolygonData que proporciona el polígono de control, es decir, el que se corresponde con el perímetro general que no contiene vértices truncados.

Figura 11. Identificación de candidatos a vértice truncado.

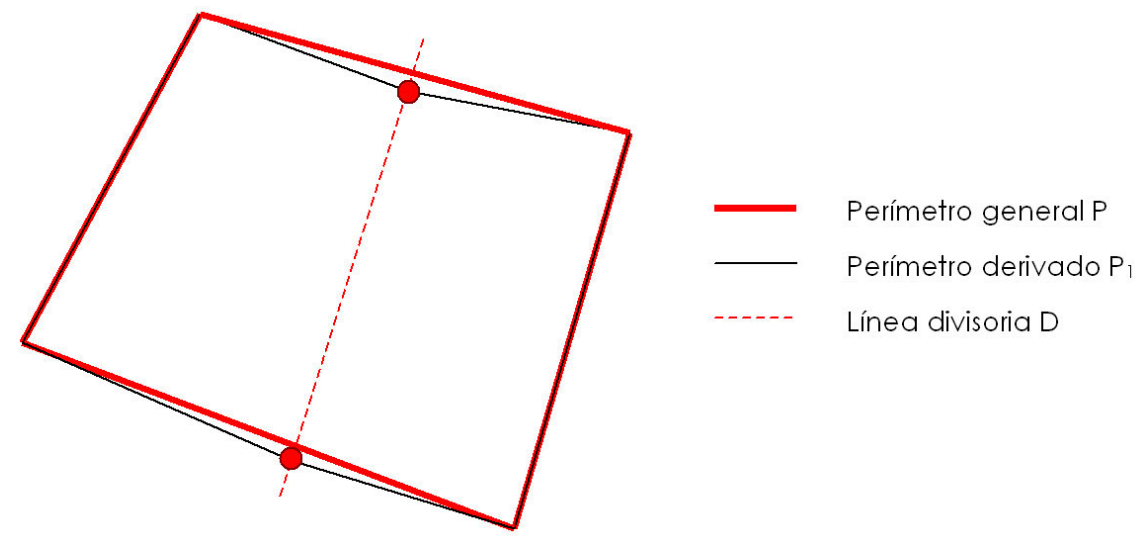

El output del método FilterPolygon es una nueva estructura de tipo PolygonFilterData que hereda los identificadores de las parcelas participantes en el grafo y en la unión de polígonos (propiedad Ids). No existe ningún requisito adicional para la validez de esta estructura excepto que los inputs sean válidos a su vez. En cualquier caso, ante un incidente durante el proceso de cálculo la estructura queda invalidada (la propiedad IsValid se asigna a false) y el error se almacena (propiedad Log). En cuanto al resultado geométrico, la propiedad Polygon de la estructura PolygonFilterData mantiene una referencia al polígono filtrado que debe coincidir en todos sus vértices con el polígono aportado por la estructura PolygonUnionData excepto en aquellos que han sido eliminados por el algoritmo responsable de decidir si un candidato a vértice truncado lo es efectivamente.

Figura 12. Diagrama UML del filtrado de vértices truncados.

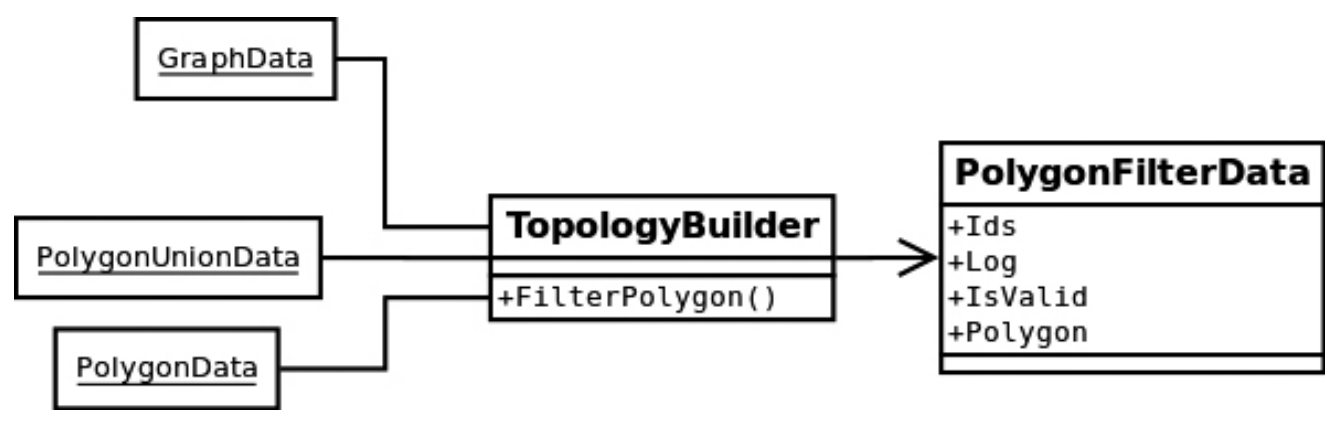

El algoritmo de filtrado para un candidato a vértice truncado se basa en la evaluación de la siguiente regla:

$$
\exists \operatorname{DIST}(c, P) \leq k
$$

En una rejilla centimétrica, $k$ es la distancia desde el centro de una celda cuadrada a cualquiera de sus esquinas, es decir, el valor de $k$ equivale a la mitad de la longitud de la hipotenusa de un triángulo rectángulo con catetos de longitud igual a $1 \mathrm{~cm}(0,7 \mathrm{~cm}$ aproximadamente). Por tanto, un candidato en la coordenada $c$ es efectivamente eliminado sólo si la distancia desde la coordenada al perímetro de control $(\operatorname{DIST}(c, P))$ es menor o igual a $k$. Esta constante define una tolerancia perfectamente asumible en el ámbito catastral. En la imagen de la Figura 18 se puede apreciar un buen ejemplo de las consecuencias de errores de este tipo. 


\subsection{Evaluación de igualdad topológica}

En el modelo de validación del Proyecto "Ramón Llull", la evaluación de igualdad topológica entre pares de polígonos es el objetivo final de todos los planes de validación vinculados a las operaciones de alteración catastral. En este contexto dos objetos de tipo Polygon se consideran topológicamente iguales cuando el predicado Relate da como resultado una matriz conforme al patrón FF2F11212 (véase la Tabla 1).

La diferencia entre la igualdad geométrica y la igualdad topológica queda ilustrada en la Figura 13, donde se observan dos geometrías que coinciden dimensional y posicionalmente, pero que difieren en cuanto al número total de vértices y su orden.

El método ComputeEquality de la clase TopologyBuilder es el responsable de evaluar la igualdad topológica a partir de un input que siempre estará constituido por un par de geometrías poligonales aportadas por sendas estructuras de validación. La evaluación de igualdad depende de la naturaleza de la operación que estemos validando, por lo que el método ComputeEquality es polimórfico. Así pues, las combinaciones de pares de estructuras aceptadas como input son:

PolygonUnionData + PolygonData en el caso de agregaciones. La primera aporta el polígono resultante de la unión de las parcelas originales en la base cartográfica. La segunda aporta el polígono propuesto como parcela agregada.

PolygonFilterData + PolygonData en el caso de segregaciones y divisiones parcelarias. La primera aporta el polígono resultante de la unión de las parcelas segregadas propuestas una vez desechados los vértices truncados. La segunda aporta el polígono de la parcela matriz en la base cartográfica.

Figura 13. Ejemplo de polígonos que coinciden topológicamente pero cuya geometría difiere.

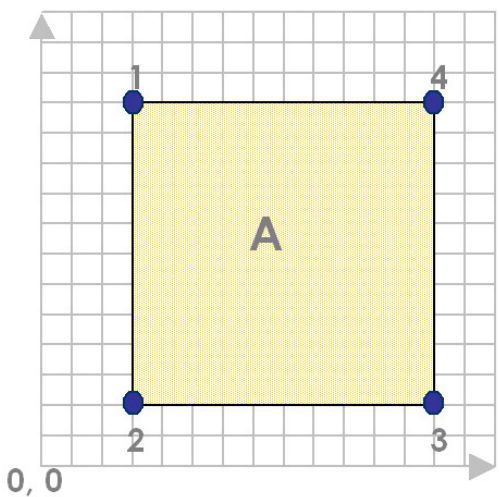

\section{$\exists$ A.Relate(B) $\in$ FF2F1 1212}

0,0

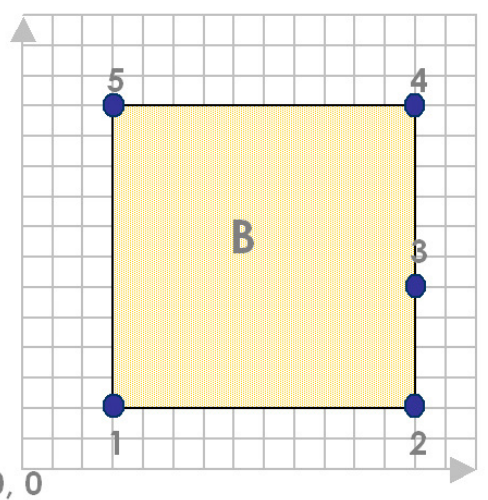

Para una segregación, una división parcelaria o un test de coincidencia el método de cálculo se basa en el predicado Relate y en la matriz patrón ya mencionada. Sin embargo, en el caso de la agregación, se utiliza el predicado predefinido EqualsExact de NetTopologySuite, es decir, se busca una coincidencia geométrica exacta ya que se considera que una agregación de parcelas no ha de introducir, si no es por causa justificada, vértices nuevos en la base cartográfica de referencia. Como se aprecia en el diagrama UML de la Figura 14, el output del método ComputeEquality es una nueva estructura de tipo EqualityData. El resultado booleano de la evaluación de igualdad topológica se registra en la propiedad IsValid, mientras que las posibles incidencias pueden recuperarse mediante la propiedad Log.

Figura 14. Diagrama UML de la evaluación de igualdad topológica.

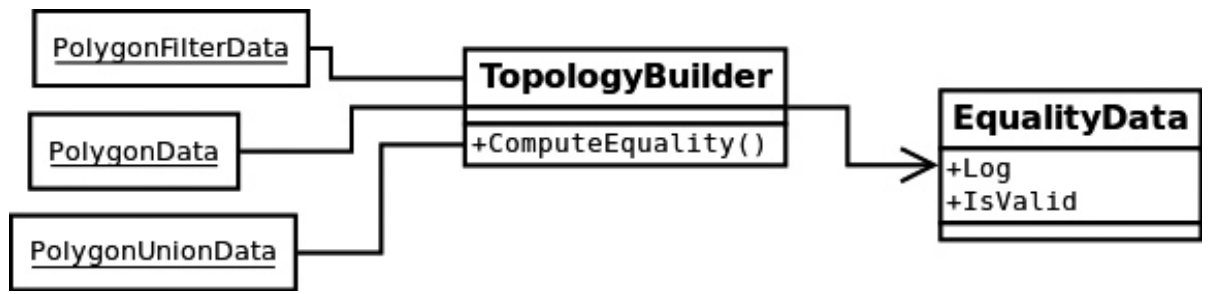




\section{PLANES DE VALIDACIÓN TOPOLÓGICA}

En el ámbito del Proyecto "Ramón Llull" el concepto de plan de validación topológica se define como una secuencia lógica de reglas de cálculo sobre el par de operandos de una alteración catastral, entendiendo por operando el perímetro general de la parcela o parcelas procedentes bien de la base cartográfica de referencia, bien del proyecto propuesto. El resultado de un plan de validación es siempre un diagnóstico favorable o desfavorable, y los escenarios que impiden o interrumpen la ejecución de la secuencia son, respectivamente, la ausencia de alguno de los operandos y la no conformidad con alguna de las reglas de cálculo.

Desde cada tipo de alteración catastral del modelo de expediente (agregación, segregación o división parcelaria) se puede ejecutar el plan de validación correspondiente y generar una instancia de la clase Validation, tal como ilustra el diagrama UML de la Figura 15.

Figura 15. Diagrama UML de los planes de validación topológica.

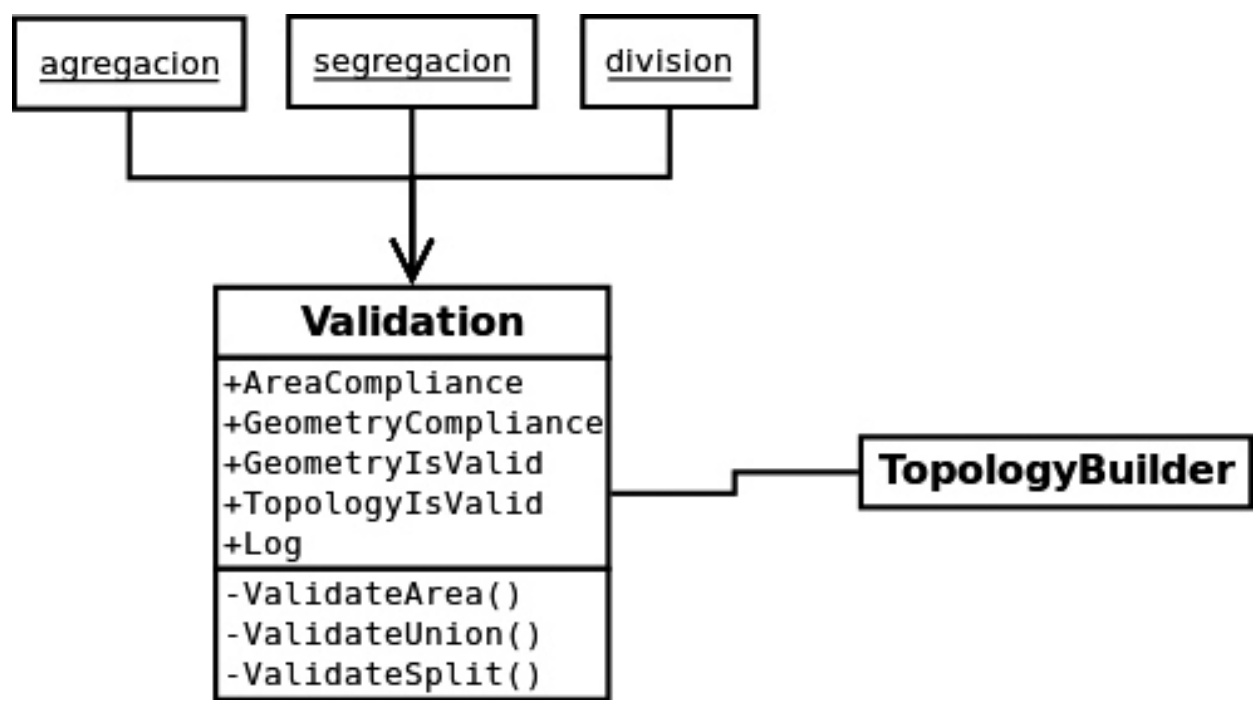

Así pues, la clase Validation encapsula la implementación de todas las secuencias posibles de validación topológica. Para certificar con el mayor grado posible de confianza la coherencia de la alteración catastral, una secuencia siempre cubre dos aspectos: el análisis comparativo de superficies (común a todas las secuencias) y el análisis de las relaciones espaciales entre los operandos (específico para cada tipo de operación).

El método ValidateArea realiza el análisis comparativo de superficies con el objetivo de almacenar en la propiedad AreaCompliance uno de cinco posibles estados: coincidencia, déficit tolerable, déficit no tolerable, exceso tolerable y exceso no tolerable. La condición general que define un diagnóstico de superficie favorable puede expresarse como:

$$
\exists|A R E A(B)-A R E A(A)|<A R E A(A) * t
$$

Donde $A$ es el operando procedente de la base cartográfica de referencia, $B$ es el operando aportado por el proyectista y $t$ es la tolerancia de desfase determinada por la constante 0,05 (porcentaje de superficie del operando A por debajo del cual ambas superficies pueden considerarse equivalentes a efectos catastrales).

El método ValidateSplit implementa el análisis de relaciones espaciales para segregaciones y divisiones parcelarias, mientras que el método ValidateUnion lo hace para agregaciones. Durante su ejecución, todos ellos asignan el valor correspondiente a las propiedades de diagnóstico topológico (GeometryIsValid, TopologyIsValid y GeometryCompliance), que, aunque hacen referencia a enumeraciones, en última instancia pueden considerarse como booleanas. Dichos métodos generan a su vez una descripción detallada de los resultados de cada paso del plan de validación, la cual se almacena en la propiedad Log. 


\subsection{Plan de validación de segregaciones a modo de ejemplo}

Una explicación pormenorizada del análisis de relaciones espaciales en el plan de validación de segregaciones ofrece un buen ejemplo del grado de complejidad que puede alcanzar la secuenciación de reglas de cálculo. Se ha escogido el enfoque de los diagramas de flujo como medio de representación puesto que permiten hacer referencia directa al modelo de objetos del motor de cálculo topológico, es decir, a las estructuras de validación de entrada y salida de cada paso de la secuencia.

La secuencia de validación de segregaciones aplica tanto a segregaciones por pares (i.e. parcela segregada y resto de finca matriz) como a divisiones parcelarias y tiene, por este orden, una fase geométrica o preproceso, y otra topológica.

Figura 16. Fase geométrica de la secuencia de validación de segregaciones.

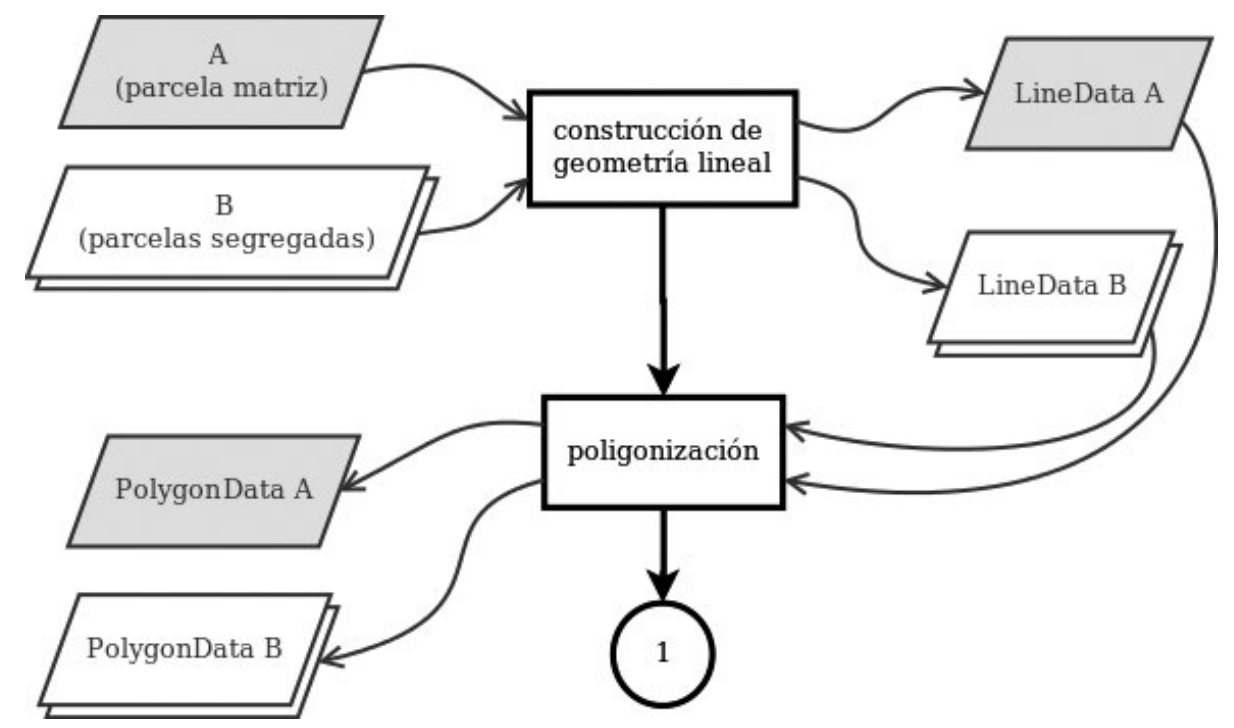

Como ilustra el diagrama de flujo de la Figura 16, los datos de partida u operandos al inicio de la fase geométrica son:

1. El operando A o perímetro general de la parcela matriz procedente de la base cartográfica de referencia.

2. El operando B o conjunto de perímetros generales de las parcelas segregadas en proyecto.

Dejando al margen el caso menos probable de inconsistencia en el contorno de la parcela matriz preexistente, el fracaso del proceso de poligonización suele estar causado por intersecciones no materializadas (líneas inacabadas o sobreacabadas que impiden el cierre del contorno) en las parcelas segregadas propuestas, fruto de una captura defectuosa de los nuevos lindes. En cualquier caso, esto implica un diagnóstico desfavorable y la devolución de los perímetros al técnico proyectista para su corrección.

La fase topológica de la secuencia de validación de segregaciones es compleja dado que cualquier fragmentación parcelaria conlleva necesariamente la construcción de nuevos vértices, tanto en el interior de la parcela matriz como, sobre todo, en su contorno exterior. El diagrama de flujo de la Figura 17 muestra todos los procesos implicados en la secuencia, los cuales revisaremos a continuación precisamente para analizar las causas que más probablemente pueden invalidar determinadas estructuras de datos de salida.

Según diversas pruebas sobre datos reales, el punto de ruptura más frecuente de esta secuencia lo encontramos en la estructura AdjacencyData, cuya evaluación fallida provoca un diagnóstico desfavorable por topología inválida. La orientación acerca de las causas la encontramos en la estructura PolygonIMData, generada en el paso anterior, que suele indicar superposiciones entre los polígonos del operando $B$ (las parcelas segregadas en proyecto). Por tanto, aunque el proceso de poligonización culmine con éxito, existe la posibilidad de superposición entre los polígonos construidos. Curiosamente, esta incoherencia se produce con mayor probabilidad si las líneas divisorias han sido tratadas mediante aplicaciones 
informáticas que están diseñadas para automatizar o asistir a los técnicos en los procesos de reparcelación, dado que en ocasiones dichas aplicaciones pueden no generar un duplicado exacto de un tramo de linde compartido por dos fincas, lo que en última instancia conduce a la formación de slave polygons. El modelo de validación topológica del Proyecto "Ramón Llull" ayuda a detectar estas situaciones y a remitir de nuevo al técnico los perímetros de las parcelas segregadas para su rectificación.

Figura 17. Fase topológica de la secuencia de validación de segregaciones.

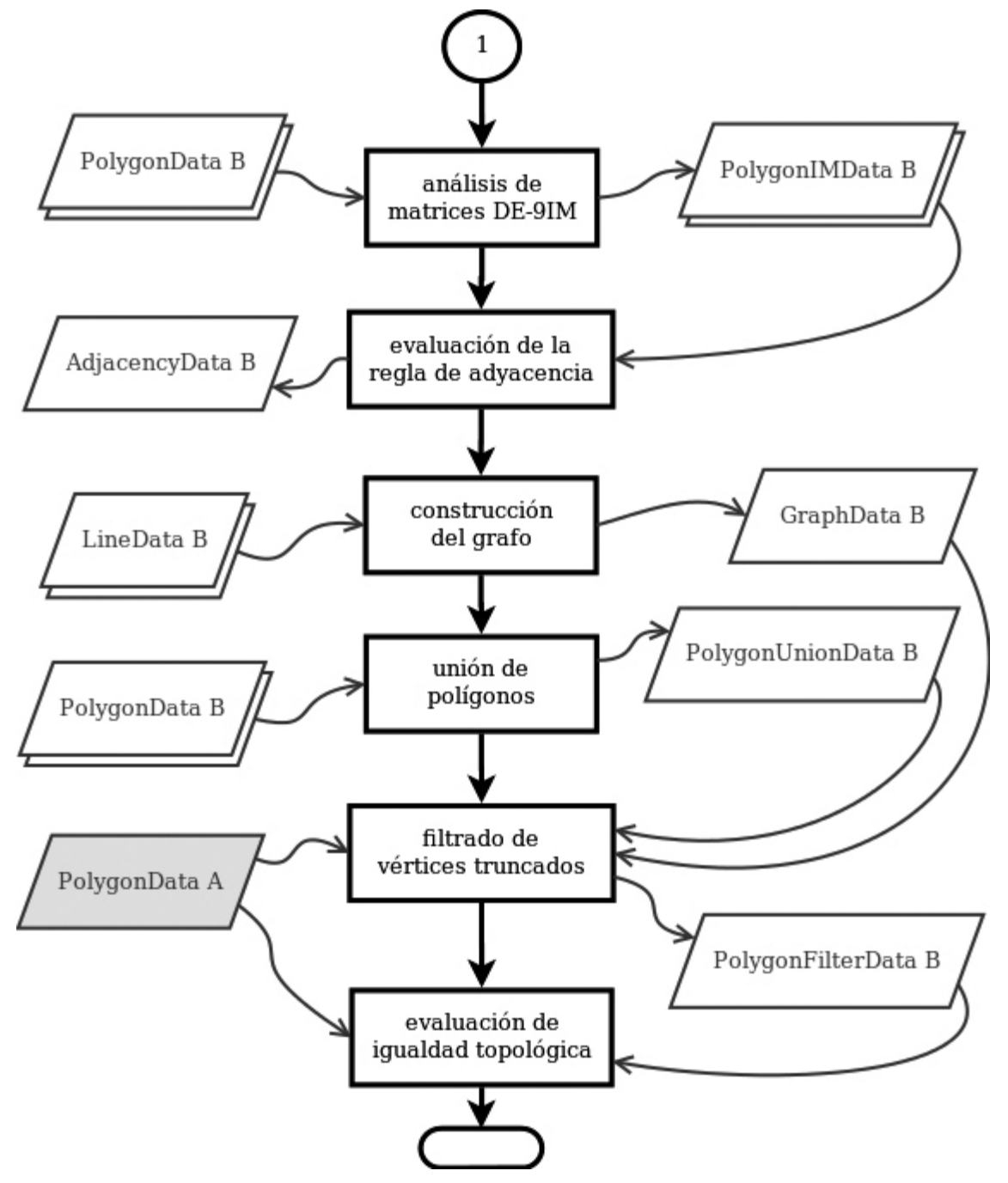

Si partimos de una estructura AdjacencyData válida, el siguiente paso de la secuencia es la construcción del grafo topológico a partir de los objetos MultiLineString que conforman los perímetros generales de las parcelas segregadas, y a los que se accede mediante la lista de estructuras LineData del operando $B$ generadas en el primer paso. A continuación se procede con la unión de polígonos de parcelas segregadas. La estructura PolygonUnionData resultante junto con la estructura GraphData del paso anterior y el polígono de la parcela matriz generado en la fase geométrica (PolygonData del operando A) constituyen el input del proceso de filtrado de vértices truncados, cuya lógica ya se ha explicado anteriormente. De esta forma obtenemos una estructura PolygonFilterData con la que culmina el tratamiento de los nuevos vértices introducidos durante el trazado de las líneas divisorias y con la que se puede afrontar el último paso de la secuencia: la evaluación de igualdad topológica entre el perímetro general de la parcela matriz (PolygonData del operando A) y el perímetro circundante de las parcelas segregadas (PolygonFilterData). Los factores que suelen determinar la falta de coincidencia entre estos dos perímetros son la traslación de vértices, un conjunto incompleto o mal trazado de parcelas segregadas o la consabida discrepancia 
entre la base cartográfica de referencia y el hipotético levantamiento de lindes que haya podido realizar el proyectista en el campo. En ausencia de estas circunstancias y ante una estructura EqualityData válida, el diagnóstico de la segregación será favorable.

Figura 18. Podemos ver un ejemplo de falsa colindancia entre los lados adyacentes de la parcela ...5534104BC... y la parcela ...5533110BC... (marcados en rojo), dando como resultado un hueco inapreciable a vista incluso de detalle de zoom. Este espacio entre polígonos se produce cuando se crean polígonos nuevos sin tener en cuenta la topología y podría dar problemas a la hora de validar una agregación entre ambas en Catastro. El análisis topológico que realiza la aplicación "Ramón Llull" detectó que las dos parcelas que el cliente proponía para la agregación no eran coincidentes y por lo tanto no debían generar un único polígono resultante.

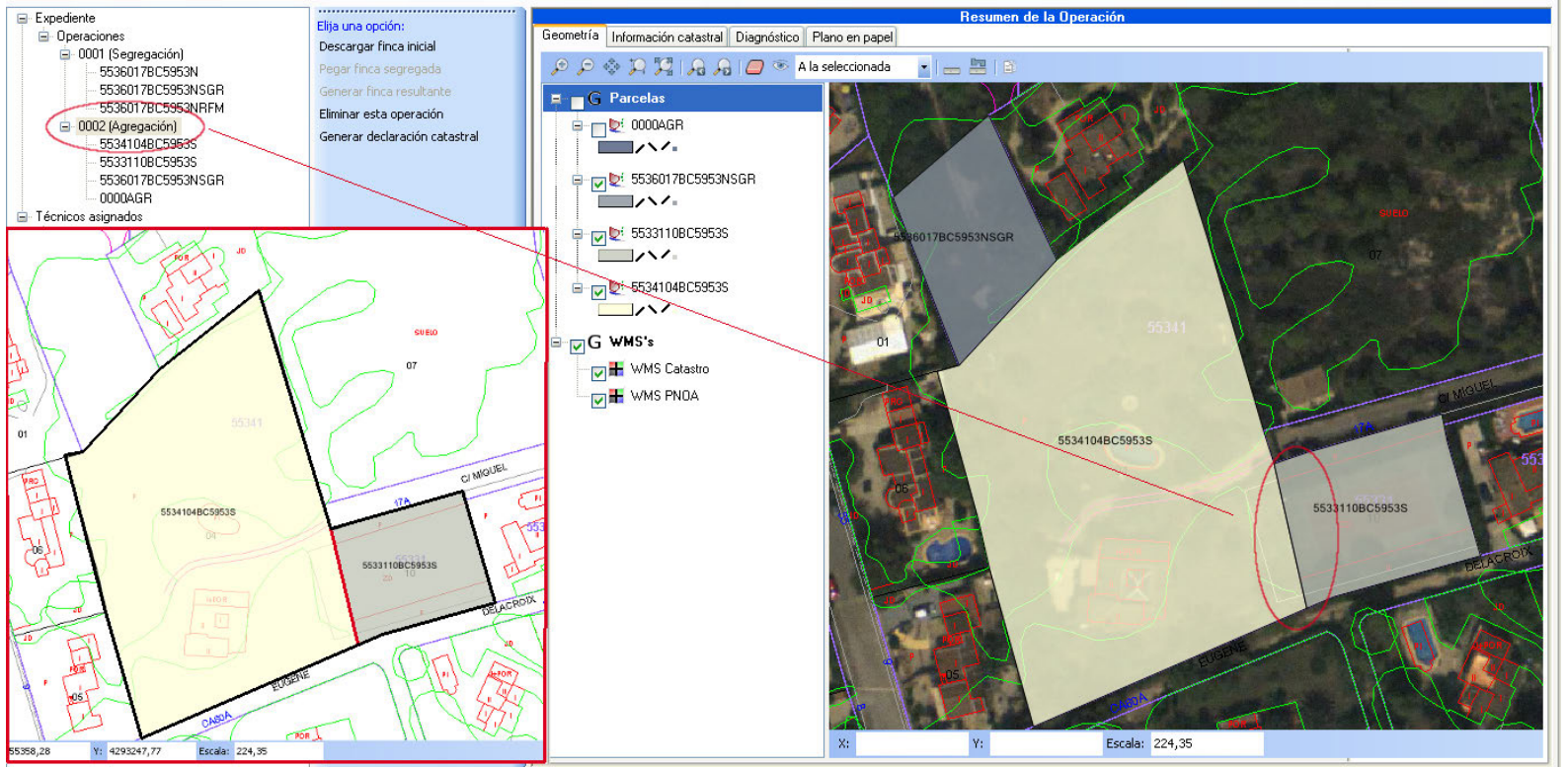

\section{CONCLUSIONES}

En el marco de la gestión y el tratamiento de las bases de datos catastrales, el Proyecto "Ramón Llull" supone un enfoque nuevo y complementario. Hasta el momento, el valor de la información espacial relativa a inmuebles se sustentaba en su utilidad de cara a la dinamización de los procedimientos tributarios. Esto ha propiciado que la base cartográfica de referencia se actualice de forma masiva y periódica en función del trabajo realizado por aquellos organismos públicos o privados con competencias tributarias que han conveniado con la Dirección General de Catastro. El fruto de este proceso es una cartografía catastral cada vez más veraz y actualizada, pero con el sesgo de gestión tributaria y no de información geográfica de "amplio espectro".

El Proyecto "Ramón Llull" aprovecha la ventaja que supone disponer al menos, de esta cartografía de calidad, para poder ir un poco más lejos y dar carta de naturaleza al valor jurídico inherente al dato geográfico que describe un bien inmueble. De esta forma, haciendo coincidir el acto jurídico que acompaña a cualquier alteración catastral con la actualización de la base cartográfica de referencia, la base de datos del Catastro ya no sólo depende de la cadencia temporal de las transacciones masivas, sino que puede reflejar los cambios en la distribución espacial de la propiedad casi en tiempo real, mediante transacciones individuales correctamente tramitadas, incluso vía electrónica. El modelo de validación topológica descrito busca precisamente otorgar el mayor grado posible de fiabilidad a este tipo de transacciones, de forma que la Dirección General de Catastro promueva esta política de actualización articulada en torno a servicios remotos.

Sin duda, el gran reto de este proyecto es su aplicabilidad, teniendo en cuenta que no es nada sencillo aunar la complejidad del proceso de este tipo de información con la facilidad de manejo por parte del usuario, tenga el perfil que tenga. En este sentido, el problema estriba en implantar soluciones basadas en Tecnologías de la Información Geográfica en entornos profesionales habituados a la gestión ofimática 
de documentación jurídica pero ajenos al tratamiento de cartografía digital y a los conceptos de bases de datos geográficas y análisis espacial. Precisamente por ello, este modelo de validación topológica proporciona al desarrollador un marco lógico suficientemente flexible como para acercar el dato geográfico al usuario sin renunciar a la posibilidad de poder representar mediante estructuras espacio-temporales casos verdaderamente complejos de alteraciones catastrales. La aplicación de escritorio desarrollada en el Laboratorio de Geomática de la Universidad de Alicante para la gestión de expedientes de alteración catastral en notarías (véanse Figuras 18 y 19), actualmente en fase experimental de explotación, emplea el modelo de validación topológica aquí presentado desde la triple perspectiva de facilidad, flexibilidad y transparencia, procurando ofrecer al usuario la información necesaria para verificar la coherencia de una alteración de una forma rápida y sencilla. Esta aplicación permite la generación sistemática de expedientes complejos, con múltiples alteraciones, facilitando el acceso aleatorio a la información cartográfica tanto a nivel de operación como de finca, así como la evaluación a demanda del diagnóstico de cada una de las operaciones, la generación de los correspondientes informes técnicos de detalle y la salida impresa de planos georreferenciados.

Figura 19. Aplicación de escritorio del Proyecto "Ramón Llull": visualización cartográfica de una operación de alteración catastral y de la ventana de diagnóstico que informa a los usuarios de la pertinencia y correción de la operación, incluida la validación topológica.

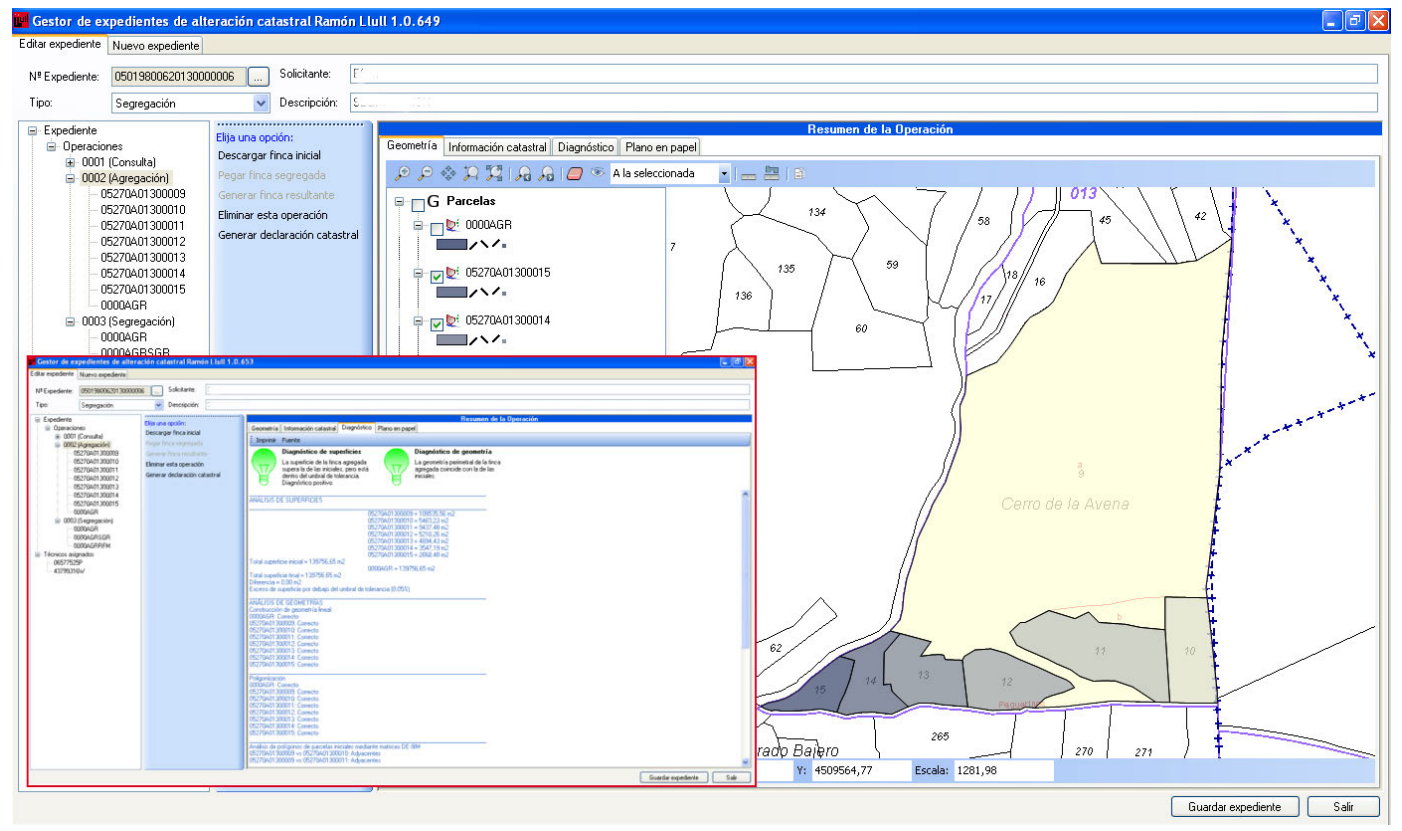

En síntesis, el modelo de validación topológica constituye el núcleo de la propuesta del Proyecto "Ramón Llull" para verificar la autenticidad física de las fincas que intervienen en una alteración catastral y otorgar mayor seguridad a los actos jurídicos que regulan las transacciones de bienes inmuebles. Su diseño está basado en una metodología rigurosa en la que han prevalecido los principios de estandarización (la normalización es una cuestión decisiva de cara a la Norma ISO19152), interoperabilidad, robustez y flexibilidad en lo relativo al esquema lógico, todo ello con vistas a la implantación de una plataforma distribuida de servicios de base de datos geográfica y capaz de tratar estructuras espacio-temporales con agilidad. Constituye una respuesta desde la investigación geográfica aplicada a las limitaciones que históricamente se vienen produciendo en el tratamiento o gestión de la información catastral por parte del usuario público, con la sinergia derivada de la doble perspectiva tecnológica (Tecnologías de la Información Geográfica) y jurídico-social (tanto humanística, como jurídica) para buscar soluciones que promuevan la coherencia, difusión y uso equitativo de este tipo de información geográfica, en la línea que en su momento marcaron iniciativas del calado e importancia del Proyecto Ensenada, SIGCA o SIGCA2.

Destacamos en este artículo la necesidad de la información cartográfica o geográfica en la gestión de bienes inmuebles y en el proceso de alteración de datos catastrales, proponemos una metodología que 
pueda superar la visión del dato geográfico como un simple dibujo de CAD, buscando su dimensión de bases de datos SIG, señalamos la importancia de trabajar con criterios topológicos que permitan obtener geometrías de calidad y cotejar la información de campo con las bases de datos geográficas oficiales, con garantías de seguridad técnica. Finalmente, resolvemos la intervención de especialistas con perfil jurídico, mediante el desarrollo de aplicaciones informáticas que hagan fácil y transparente el empleo de esta información por parte de usuarios no expertos en Cartografía, lo que permite ofrecer también todas las garantías jurídicas necesarias, en el momento más adecuado, al otorgamiento de la escritura pública que recoge esta alteración y a instancias de los ciudadanos y propietarios interesados.

\section{BIBLIOGRAFÍA}

ARROYO ILERA, F. y CAMARERO BULLÓN, C. (1992): "La Geografía histórica en España", en REAL SOCIEDAD GEOGRÁFICA; ASOCIACIÓN DE GEÓGRAFOS ESPAÑOLES: La Geografía en España (1970-1990). Aportación española al XXVII Congreso de la Unión Geográfica Internacional, Fundación BBV, Madrid.

BENENSON, I., ARONOVICH, S. y NOAM, S. (2005): "Let's talk objects: generic methodology for urban high-resolution simulation", Computers, Environment and Urban Systems, num. 29, pp. 425-453, Elsevier.

BOOCH, G., RUMBAUGH, J. y JACOBSON, I. (1999): El Lenguaje Unificado de Modelado, Addison Wesley, Reading, Massachusets.

BOSQUE SENDRA, J. (1992): Sistemas de Información Geográfica, Rialp, Madrid.

BURROUGH, P. (1989): Principles of Geographical Information Systems for land resources assessment, Oxford University Press, Oxford.

BUZAI, G. D. (1999): "Geografía Global. El paradigma geotecnológico y el espacio interdisciplinario en la interpretación del mundo del siglo XXI.", Anais GIS Brasil 99. V Congresso e Feira para usuarios de geoprocessamento da America Latina, Sagres Editora Ltda., San Salvador de Bahía.

CHUVIECO, E., BOSQUE, J., PONS, X., CONESA, C., SANTOS, J.M., GUTIÉRREZ-PUEBLA, J., SALADO, M.J., MARTÍN, M. P., RIVA, J., OJEDA, J. y PRADOS, M.J. (2005): “¿Son las Tecnologías de la Información Geográfica (TIG) parte del núcleo de la Geografía?", Boletín de la Asociación de Geógrafos Españoles, num. 40, pp. 35-55, Madrid.

COMAS, D. y RUIZ, E. (1993): Fundamentos de los Sistemas de Información Geográfica, Ariel, Barcelona.

DEVIS BOTELlA, R. (1993): Programación Orientada a Objetos en C++, Paraninfo, Madrid.

DÍAZ DÍAZ, E.:

- (2013): "Alteración catastral telemática e inscripción registral", Revista El Notario del Siglo XXI. Revista del Ilustre Colegio Notarial de Madrid. $n^{\circ} 51$. http://www.elnotario.es/index.php/practicajuridica/3551-alteracion-catastral-telematica-e-inscripcion-registral

- (2012): "Claves de la interoperabilidad jurídica de la información geográfica" Blog de la Infraestructura de Datos Espacial de España. Instituto Geográfico Nacional. Madrid: http://blogidee.blogspot.com.es/2012/06/claves-de-la-interoperabilidad-juridica.html

- (2012): "Interoperabilidad jurídica de la geoinformación" en las Jornadas de la Infraestructura de Datos Espacial de España 2012. Instituto Geográfico Nacional. Madrid: http://www.idee.es/web/guest/ jornadas

ESPIAGO, J. (2007): Prólogo a Requejo Liberal, Descripción geográfica de las fincas en el Registro de la Propiedad (Geo-Base), Lex Nova. Valladolid.

GARCÍA RAMÓN, M. D., NOGUÉ I FONT, J. y ALBET I MAS, A. (1992): La práctica de la Geografía en España, Oikos-Tau, Barcelona.

GIL OLCINA, A. (1992): "El mundo rural", en REAL SOCIEDAD GEOGRÁFICA; ASOCIACIÓN DE GEÓGRAFOS ESPAÑOLES: La Geografía en España (1970-1990). Aportación española al XXVII Congreso de la Unión Geográfica Internacional, Fundación BBV, Madrid. 
HERNÁNDEZ ORALLO, E. (2002): "El lenguaje Unificado de Modelado", Manual Formativo ACTA, num. 26, pp. 69-74, Autores Científico-Técnicos y Académicos, Madrid.

HOWE, D. R. (1989): Data Analysys for Data Base Design, 2nd edition, Edward Arnold, Londres.

HUXHOLD, W. E. (1991): An Introduction to Urban Geographic Information Systems, Oxford University Press, Oxford.

JIMÉNEZ CLAR, A. J.:

- (2010): "La coordinación catastro-registro desde una perspectiva internacional", Revista El Notario del Siglo XXI. Revista del Ilustre Colegio Notarial de Madrid. $n^{\circ} 39$. http://www.elnotario.es/ index.php/100-hemeroteca/revistas/revista-39/667-la-coordinacion-catastro-registro-desde-unaperspectiva-internacional-0-48886309050849

- (2010): "La información territorial: ¿es posible la unificación del Catastro y del Registro?, Revista El Notario del Siglo XXI. Revista del Ilustre Colegio Notarial de Madrid. $n^{0} 32$. http://www.elnotario. es/index.php/107-hemeroteca/revistas/revista-32/1119-la-informacion-territorial-es-posible-launificacion-del-catastro-y-del-registro-0-3271639722652398

JIMENEZ CLAR, A.J. y RAMÓN MORTE, A. (2008): Hoja de Ruta del Proyecto Ramón Llull, Dpto. de Derecho Civil y Laboratorio de Geomática del Instituto Universitario de Geografía, Universidad de Alicante (inédito).

KEMP, Z. y KOWALCZYYK, A. (1994): "Incorporating the temporal dimension in a GIS", en WORBOYS, M. F. (ed.): Innovations in GIS: Selected Papers from the First National Conference on GIS Research UK, pp. 89-104, Taylor \& Francis, Londres.

LLORENS COBOS, F., MIRA MARTINEZ, J.M., NAVARRO CARRIÓN, J.T. y RAMÓN MORTE, A. (2007): "Proyecto Ramón Llull: sistema de alteraciones catastrales para las notarías de la Comunidad Valenciana", Comunicaciones de las I Jornadas de SIG Libre, SIGTE, Universitat de Girona.

SÁNCHEZ JORDÁN, M.E. (2012): "Nuevas técnicas de identificación de las fincas: su aplicación al deslinde". Anuario de Derecho Civil. Núm. LXV-III, Julio 2012. pp. 1075-1106. Universidad de La Laguna. http://vlex.com/source/anuario-derecho-civil-1714/issue nbr/\%23LXV-III

MAGUIRE, D.J, GOODCHILD, M.F. y RHIND, D.W. (coords.) (1991): Geographical Information Systems: Principles and Applications, Longman, Londres.

MEYER, B. (1988): Object-Oriented Software Construction, Prentice Hall, Nueva Jersey.

OGC (2006): OpenGIS Implementation Specification for Geographic information - Simple Feature Access - Part 1: Common architecture, ref. OGC 06-103r3, Open Geospatial Consortium Inc.

TORRENS, P. M. y BENENSON, I.:

- (2005a): "Geographic Automata Systems", International journal of Geographical Information Systems, vol. 19, num. 4, pp. 385-412, Taylor \& Francis, Londres.

- (2005b): "A Minimal Prototype for Integrating GIS and Geographic Simulation through Geographic Automata Systems", en ATKINSON, P. M. y otros (eds.): GeoDynamics, pp. 347-367, CRC Press, Londres.

UNWIN, D. (1981): Introductory Spatial Analysis, Methuen, London.

UREÑA CÁMARA. M. A., GARCíA BALBOA, J.L. y ARIZA LÓPEZ, J. (2010): “Análisis de la propuesta ISO 19152 (Land Administration Domain Model)" en Actas del I Congreso Internacional de Catastro Unificado y Multipropósito. Cádiz. 2010.

VIVID SOLUTIONS (2003): JTS Topology Suite Technical Specifications, Vivid Solutions.

VÁZQUEZ ASENJO, Ó.:

- (2009): La información territorial asociada a las bases gráficas registrales, Tirant lo blanch, Valencia.

- (2013): Coordinacion entre el catastro y el registro de la propiedad. Tirant lo blanch, Valencia. 
W3C (2006): Extensible Markup Language (XML) 1.1 (Second Edition), World Wide Web Consortium.

WACHOWICZ, M. y HEALEY, R. G. (1994): "Towards temporality in GIS", en WORBOYS, M. F. (ed.): Innovations in GIS: Selected Papers from the First National Conference on GIS Research UK, pp. 105-118, Taylor \& Francis, Londres.

WACHOWICZ, M. (1999): Objet-Oriented Design for Temporal GIS, Taylor \& Francis, Londres.

WIRTH, N. (1980): Algoritmos + estructuras de datos = programas, Ediciones del Castillo, Madrid.

WORBOYS, M. F., HEARNSHOW, H. M. y MAGUIRE, D. J. (1990): "Object-oriented modelling for spatial databases", International journal of Geographical Information Systems, vol. 4, num. 4, pp. 369-383, Taylor \& Francis, Londres.

YEARSLEY, C. y otros (1994): "Computational support for spatial information systems: models and algorithms", en WORBOYS, M. F. (ed.): Innovations in GIS: Selected Papers from the First National Conference on GIS Research UK, pp. 75-88, Taylor \& Francis, Londres.

ZHU, X. y HEALEY, R. G. (1992): "Towards intelligent spatial decision support: integrating geographical information systems and experts systems", Proceedings of GIS/LIS '92, vol. 2, pp. 877-886, ACSM/ ASPRS/AAG/URISA/AM-FM, San José (California).

ZHU, X., ASPINALL, R. J. y HEALEY, R. G. (1996): "ILUDDS: A knowledge-based spatial decision support system for strategic land use planning", Computer and Electronics in Agriculture, num. 15, pp. 279-301, Elsevier.

ZOIDO NARANJO, F. (2001): "Relaciones entre formación y dedicación profesional en la geografía española", Documents d'Anàlisi Geogràfica, num. 39, pp. 37-56, Barcelona. 